\title{
Hexagonal boron nitride on transition metal surfaces
}

\author{
Jaime Gómez Díaz · Yun Ding • Ralph Koitz • \\ Ari P. Seitsonen • Marcella Iannuzzi • \\ Jürg Hutter
}

Received: 31 October 2012/ Accepted: 1 February 2013/Published online: 27 February 2013

(c) The Author(s) 2013. This article is published with open access at Springerlink.com

\begin{abstract}
We validate a computational setup based on density functional theory to investigate hexagonal boron nitride $(h$-BN) monolayers grown on different transition metals exposing hexagonal surfaces. An extended assessment of our approach for the characterization of the geometrical and electronic structure of such systems is performed. Due to the lattice mismatch with the substrate, the monolayers can form Moiré-type superstructures with very long periodicities on the surface. Thus, proper models of these interfaces require very large simulation cells (more than 1,000 atoms) and an accurate description of interactions that are modulated with the specific registry of $h$-BN on the metal. We demonstrate that efficient and accurate calculations can be performed in such large systems using Gaussian basis sets and dispersion corrections to the (semi-)local density functionals. Four different metallic substrates, $\mathrm{Rh}(111), \mathrm{Ru}(0001), \mathrm{Cu}(111)$, and $\mathrm{Ni}(111)$, are explicitly considered, and the results are compared with previous experimental and computational studies.
\end{abstract}

Keywords Density functional theory - Nanomesh .

Boron nitride $\cdot$ Interfaces $\cdot$ Metal surfaces

\section{Introduction}

Precise positioning and the control of interactions of individual molecules or small assemblies of atoms are of prime importance in the field of nano-devices. A promising way

J. Gómez Díaz: Deceased.

J. Gómez Díaz · Y. Ding · R. Koitz · A. P. Seitsonen

M. Iannuzzi · J. Hutter $(\bowtie)$

Physical Chemistry Institute, University of Zürich,

Winterthurerstrasse 190, 8057 Zurich, Switzerland

e-mail: Hutter@pci.uzh.ch to obtain well-defined arrangements on a large scale is through template surfaces. These show a variation of the interaction strength on a nanometer scale. Optimally, the template will not interact too strongly with the nanostructures and should affect only weakly their electronic properties. Such functionalized surfaces have many possible applications in chemistry and biology and are essential in nano-electronics.

Monolayers of hexagonal boron nitride ( $h$-BN) [1], as well as the isoelectronic carbon structure, graphene (gr), grown on transition metal (TM) surfaces have received much interest as possible templates for use in nano-devices. Chemical vapour deposition of precursor molecules, e.g., borazine in the case of $h$-BN, on a hot metallic surface leads to a spontaneous formation of the uniform epitaxial monolayers. Originally, the preparation of a single layer of $h$-BN was achieved on the $\mathrm{Rh}(111)$ surface $[1,2]$, but recently similar structures have been grown on $\mathrm{Ru}(0001)$ [3-5], Pt(111) [4, 6, 7], Ni(111) [8-10], $\mathrm{Cu}(111)$ [10], $\operatorname{Pd}(111)$ [11], and $\operatorname{Ag}(111)$ [12]. One-dimensional structures of $h$-BN have been produced using the same procedure on $\operatorname{Cr}(110)$ [13], $\operatorname{Fe}(110)$ [14], and $\operatorname{Mo}(110)$ [15]. Using similar recipes, graphene structures on most of these TM surfaces have also been obtained [5, 16].

As the monolayer is adsorbed, its properties can become periodically modulated to form an extended superstructure. An important motivation of this work is to investigate the properties of such superstructures and the conditions under which they can be obtained. The long-range structure of the monolayer depends strongly on a series of factors: the mismatch $\alpha$ in the unit cell length $l$ of the free-standing monolayer and the TM surface,

$\alpha=\frac{l_{\mathrm{BN}}-l_{\mathrm{TM}}}{l_{\mathrm{TM}}}$, 
the distance-dependence of the $h$-BN to metal interaction, and the deformation energy, both in- and out-of-plane, of the monolayer. In $h$-BN/Rh(111), the balance between lattice mismatch $\alpha$ of $-7.0 \%$ and quite strong rhodiumnitrogen interaction leads to a strongly corrugated structure with a periodicity of $3.22 \mathrm{~nm}$, the so-called nanomesh [2]. The highly regular hexagonal arrangement corresponds to a coincidence lattice of $13 \times 13 h$-BN on $12 \times 12 \mathrm{Rh}$ unit cells. The adsorbate is characterized by "pores" of about $2 \mathrm{~nm}$ diameter that strongly interact with the metal and elevated regions, where the interaction with the metal is weak, that form the connected "wire" network. The strong variation in bonding leads to a corresponding variation of the electrostatic potential above the monolayer that is responsible for trapping molecules [17]. The $h$-BN/ $\mathrm{Rh}(111)$ nanomesh is a very stable structure that withstands temperatures of $1,000 \mathrm{~K}$ and can be exposed to liquids without losing its properties. Reversibility of the modification of the structure using atomic hydrogen has also been demonstrated [18]. Similar nanomesh structures have been found on $\operatorname{Ru}(0001), \operatorname{Pd}(111)$, and $\operatorname{Pt}(111)$, which present comparable or even larger lattice constant mismatches. On other metals, like $\mathrm{Ag}$, with a weak interaction and smaller differences in the lattice constants, commensurate structures with no preferred relative orientation $[9,12]$ have been observed.

Density functional theory (DFT)-based electronic structure calculations have been instrumental in the understanding of the structure and properties of the $h$-BN and gr nanomeshes [2, 5, 12, 17, 19]. Experimental techniques applied to characterize the nanomesh, i.e., scanning tunnelling microscopy (STM) and spectroscopy (STS), surface X-ray diffraction (SXRD), ultraviolet (UV), and $\mathrm{X}$-ray photoemission spectroscopy (UPS, XPS), as well as $\mathrm{X}$-ray absorption spectroscopy (XAS), all profit in interpretation from independent simulations. Previous simulations primarily used DFT with the generalized gradient approximation (GGA) and either the linearized augmented plane wave (LAPW) method [19-24] or a plane wave (PW) basis set [25-28].

We have developed an approach to investigate nanomeshes based on the Gaussian and plane wave (GPW) [29] formalism: A localized Gaussian basis positioned at each atom is used to expand the Kohn-Sham orbitals and a PW basis to describe the electron density to facilitate the calculation of the Coulomb interactions. This formalism leads to a very efficient description of the orbitals that can make use of locality in large systems and at the same time allows for the solution of the Poisson equation within linear time (in system size) [30]. By adding an empirical pair potential to account for long-range van der Waals (vdW) forces $[31,32]$ to a carefully chosen GGA functional, we achieve a balanced description of all important parts of the system energy. We will first show that the chosen model parameters give a faithful description of important properties of the individual systems, namely bulk and surfaces of metals and $h$-BN. We will then characterize four $h$-BN/ TM systems (TM: $\mathrm{Rh}, \mathrm{Ru}, \mathrm{Cu}$, and $\mathrm{Ni}$ ), thereby also providing an overview of earlier calculations and experimental data.

\section{Computational details and methods of analysis}

Calculations are performed using Kohn-Sham DFT [33, 34] within the GPW formalism as implemented in the Quickstep module in the CP2K program package [35]. Dual-space pseudopotentials [36-38] are used to describe the interaction of valence electrons with atomic cores. The pseudopotentials for boron and nitrogen assume 3 and 5 valence electrons, respectively. The atomic cores of the metals are described by either a large- or medium-size core pseudopotential. In particular, for $\mathrm{Rh}$ we use potentials with 9 or 17 explicit valence electrons (q9, q17), for Ru 8 or 16 (q8, q16), 18 for $\mathrm{Ni}$ (q18), and 11 for $\mathrm{Cu}$ (q11). The PW energy cutoff for the expansion of the density is set at $500 \mathrm{Ry}$. If not stated otherwise, the Brillouin zone is sampled only at the $\Gamma$ point. Exchange and correlation contributions are calculated with the Perdew-Burke-Ernzerhof (PBE) [39, 40] and revised PBE (revPBE) [41] GGA exchange-correlation (XC) functionals. The latter is used together with the corrections for the long-range dispersion interactions, computed using either the DFT-D2 [31] or DFT-D3 formalisms [32], that are missing in GGA's. The XC functional and its derivative are calculated on the same uniform density grid that is used for the Hartree energy and defined by the choice of the PW energy cutoff. In order to improve the accuracy and reduce numerical noise in the evaluation of the XC terms, a nearest neighbour smoothing procedure is employed. More details about these techniques are illustrated in Ref. [30]. The Fermi-Dirac smearing of occupation numbers with a $300 \mathrm{~K}$ electronic temperature is used in all calculations. Broyden density mixing [42] is used to facilitate smooth convergence within a reasonable number of iterations. Periodic boundary conditions are applied in all calculations. In calculations with slab-like models, interactions with periodic images in the direction perpendicular to the exposed surface are avoided by adding about $20 \AA$ of vacuum space above the slab.

The optimized structures of $h$-BN on the different metallic substrates are characterized in terms of structural parameters, like buckling $b_{\mathrm{BN}}$ (height difference of neighbouring $\mathrm{B}$ and $\mathrm{N}$ ), global corrugation $\Delta h_{\mathrm{B}, \mathrm{N}}$ (min-tomax height difference of $\mathrm{B}$ and $\mathrm{N}$ sublattices, respectively) and minimum distance of $\mathrm{B}$ or $\mathrm{N}$ from the $\mathrm{TM} d_{\min }(\mathrm{B}, \mathrm{N})$. 
The strength of the interaction between TM and $h$-BN is evaluated as

$E_{\mathrm{int}}=\left[E_{\mathrm{tot}}-\left(E_{\mathrm{slab}}^{f}+E_{h-\mathrm{BN}}^{f}\right)\right] / N_{\mathrm{BN}}$.

where $E_{\mathrm{tot}}$ is the energy of the full system, $E_{\text {slab }}^{f}$ and $E_{h \text {-BN }}^{f}$ are the energies of metallic slab and $h$-BN overlayer, respectively, considered individually and with the same coordinates as in the full system (without relaxing them), and $N_{\mathrm{BN}}$ is the number of the BN pairs in the full system. The adsorption energy

$E_{\mathrm{ads}}=\left[E_{\mathrm{tot}}-\left(E_{\mathrm{slab}}^{o}+E_{h-\mathrm{BN}}^{o}\right)\right] / N_{\mathrm{BN}}$

is, instead, calculated with respect to the optimized freestanding $h$-BN and the optimized bare metallic slab.

The effects on the electronic structure due to the interaction between the TM and $h$-BN is investigated by the calculation of charge density differences between the full systems and its constituents (slab and $h$-BN) at the geometry of the combined system. The density of states projected on selected subsets of atoms (PDOS) and the representation of the electronic structure through maximally localized Wannier functions (MLWF) [43] are also useful descriptors of the electronic structure properties. The Tersoff-Hamann approximation [44, 45] in STM simulations is used to yield the iso-current topography above the nanomesh. Given a bias potential $V_{b}$ (often a fraction of $\mathrm{eV}$ ), we determine the isocurrent surface as the set of grid points where

$n_{b}(z) e^{-2 k R_{0} \sqrt{\Phi(z)}}=$ const.

In this expression, $z$ is the height above the slab, $n_{b}$ the electron density due to the states with energies within $V_{b}$ from the Fermi energy, $\Phi(z)$ the local work function, $R_{0}$ an estimate of the tip curvature radius, and $k=\sqrt{2 m_{e}} / \hbar$. The constant value is set to the value of this expression at an arbitrary point laterally, in the present case at the centre of the cell, and vertically at the height $z$ where the projected density is equal to $10^{-6}$ electrons $/ \AA^{3}$, with $R_{0}=2 \AA$.

\section{Assessment of the computational setup}

Atomic basis sets, pseudopotentials and the employed density functional are the most important parameters for the model used in the nanomesh calculations. Basis sets and pseudopotentials have to be chosen carefully to find a balance between accuracy, transferability and computational efficiency. The largest systems will have a size of several thousand atoms and tens of thousands of electrons, and any justified reduction in the basis and the number of explicitly treated electrons will result in huge gains in computer time.
The Gaussian basis sets chosen for this type of application are of the molecularly optimized type [46] (Molopt basis). These basis sets are generally contracted and have a single set of exponents for all angular quantum numbers (family basis). The full contraction avoids single functions of diffuse character and results in well-conditioned overlap matrices for all kinds of systems. The basis sets include 4 primitives (B), 5 primitives $(\mathrm{N}), 6$ primitives $(\mathrm{Cu}, \mathrm{Ni}$, and $\mathrm{Rh}$ ), and 7 or 6 primitives (Ru) of $s, p, d$, and $f$ type functions. The primitives are contracted to a small number of basis functions, and we denote the basis sets with [nlijkl], where $i, j, k$, and $l$ are the number of contractions of the $s, p, d$, and $f$ type and $n$ is the number of primitives. The exponents and contraction coefficients have been globally optimized with respect to the total energy of a small set of reference molecules. The basis sets used are for $\mathrm{B}$ [4|221], N [5|221], Cu [6|2221], and Ni [6|3221]. For Rh, either the [6/212] and [6/2221] basis sets with the large core pseudopotential (9 electrons, q9) or the [613221] basis with 17 electrons (q17) are employed; for $\mathrm{Ru}$, either the [6/212] basis with 8 electrons (q8) or the [7/3221] with the q16 pseudopotential (q16).

Core electrons are described by dual-space pseudopotentials [36-38]. The potentials have been optimized in atomic calculations using the PBE [39, 40] functional. It is expected that the potentials perform equally well for the closely related revised PBE [41] functional.

\subsection{Metals}

In order to assess the computational setup, we calculated a series of properties of bulk metals and corresponding relevant surfaces and compared them with experiments and published DFT results. Table 1 shows results for the lattice constants calculated for the four metals considered. Results for the revised PBE functional and our chosen basis sets and pseudopotentials are compared to PW calculations with the same pseudopotentials ("PW-PP") [47-49], PW calculations using the projector-augmented wave (PAW) method [50] using the Quantum ESPRESSO code [49], and the experimental value. The effect of adding the empirical vdW corrections to the DFT energy is also shown.

With the revPBE functional, the optimized lattice constants are consistently larger than with the PBE functional. When either of the empirical vdW potentials is added, the lattice constants become smaller, as expected. The Molopt basis set calculations usually result in slightly smaller lattice constants when compared to PW calculations using the same pseudopotential. The only exception is Rh where we find a larger value. The contraction induced by the vdW potentials is typically $1.5 \%$ and slightly larger for the D2 than for the D3 correction. However, in copper the effect is reversed. The final lattice constant calculated with GPW 
Table 1 Optimized lattice constants $[\AA]$
GPW calculations use

$8 \times 8 \times 8$ unit cells. PW-PP and PW-PAW calculations involve one unit cell and are converged with respect to $k$-points and PW cutoff. Optimizations for $\mathrm{Ru}$ used a constant $c / a$ ratio

\begin{tabular}{|c|c|c|c|c|c|c|c|c|}
\hline & & & & PBE & revPBE & revPBE+D2 & revPBE+D3 & Experiments \\
\hline \multirow[t]{3}{*}{$\mathrm{Ni}$} & \multirow[t]{3}{*}{$\mathrm{q} 18$} & \multicolumn{2}{|l|}{ [6|3221] } & 3.479 & 3.501 & 3.429 & 3.440 & $3.5171[51]$ \\
\hline & & \multicolumn{2}{|l|}{ PW-PP } & 3.522 & 3.548 & 3.465 & & \\
\hline & & \multicolumn{2}{|l|}{$\begin{array}{l}\text { PW- } \\
\text { PAW }\end{array}$} & 3.520 & 3.546 & 3.465 & & \\
\hline \multirow[t]{3}{*}{$\mathrm{Cu}$} & \multirow[t]{3}{*}{$\mathrm{q} 11$} & \multicolumn{2}{|l|}{ [6|2221] } & 3.620 & 3.640 & 3.558 & 3.539 & 3.6149 [52] \\
\hline & & \multicolumn{2}{|l|}{ PW-PP } & 3.648 & 3.673 & 3.584 & & \\
\hline & & \multicolumn{2}{|l|}{$\begin{array}{l}\text { PW- } \\
\text { PAW }\end{array}$} & 3.644 & 3.676 & 3.589 & & \\
\hline \multirow[t]{4}{*}{$\mathrm{Ru}$} & \multirow[t]{4}{*}{$\mathrm{q} 16$} & \multirow[t]{2}{*}{ [7|3221] } & $a$ & 2.718 & 2.730 & 2.685 & 2.700 & 2.7059 [53] \\
\hline & & & $c$ & 4.292 & 4.311 & 4.240 & 4.265 & 4.2815 [53] \\
\hline & & \multirow[t]{2}{*}{ PW-PP } & $a$ & 2.759 & 2.769 & & & \\
\hline & & & $c$ & 4.366 & 4.382 & & & \\
\hline \multirow[t]{4}{*}{$\mathrm{Rh}$} & \multirow[t]{2}{*}{$\mathrm{q} 17$} & \multicolumn{2}{|l|}{ [6|3221] } & 3.847 & 3.864 & 3.796 & 3.804 & $3.803[54]$ \\
\hline & & \multicolumn{2}{|l|}{ PW-PP } & 3.834 & 3.851 & 3.781 & & \\
\hline & \multirow[t]{2}{*}{ q9 } & \multicolumn{2}{|l|}{ PW-PP } & 3.860 & 3.869 & 3.802 & & \\
\hline & & \multicolumn{2}{|l|}{$\begin{array}{l}\text { PW- } \\
\text { PAW }\end{array}$} & 3.843 & 3.854 & 3.785 & & \\
\hline
\end{tabular}

setups. For this comparison, the revPBE+D2 approach has been selected. The results are in reasonably good agreement, thus validating the use of "reduced size" setups for

Table 2 Bulk modulus B [GPa], surface energy $\Phi\left[\mathrm{eV} / \AA^{2}\right]$, and work function $\Phi[\mathrm{eV}]$ in the studied metal system

\begin{tabular}{lllllll}
\hline & Method & Functional & Cell & B & $\phi$ & $\Phi$ \\
\hline Ni & GPW & revPBE & exp. & 227 & 0.082 & 4.83 \\
& & revPBE+D3 & exp. & 285 & 0.165 & 4.81 \\
& PW-PP & revPBE & exp. & & 0.106 & 4.87 \\
& PW- & revPBE & exp. & & 0.109 & 4.92 \\
& PAW & revPBE+D2 & exp. & & 0.174 & 4.90 \\
& exp & & & 186 & $0.149,0.153$ & 5.35 \\
Cu & GPW & revPBE & exp. & 149 & 0.073 & 4.68 \\
& & revPBE+D3 & exp. & 203 & 0.136 & 4.69 \\
& PW- & revPBE & exp. & & 0.068 & 4.81 \\
& PAW & revPBE+D2 & exp. & & 0.126 & 4.80 \\
& exp & & & 137 & $0.112,0.114$ & $4.98,4.92$ \\
Ru & GPW & revPBE & opt. & 307 & & \\
& & revPBE+D2 & opt. & 323 & 0.225 & 5.01 \\
& & revPBE+D3 & opt. & 345 & & \\
& exp & & & 311 & 0.190 & 4.47 \\
Rh & GPW & revPBE & opt. & 235 & & \\
& & revPBE+D2 & opt. & 250 & 0.178 & 5.13 \\
& revPBE+D3 & opt. & 267 & & 5.12 \\
& PW- & revPBE & opt. & 238 & 0.124 & 5.01 \\
& PAW & revPBE+D2 & opt. & 256 & 0.206 & 5.02 \\
\hline & & & 270 & $0.166,0.169$ & 5.0 \\
\hline
\end{tabular}

The experimental values (B from Ref. [55] except Ru [57], $\phi$ from Ref. [57] and $\Phi$ from Ref. [58]) are shown in brackets. Computational parameters are the same used for the optimization of the lattice constants reported in Table 1 
Table 3 Properties of Ru and Rh calculated with different basis sets and pseudopotentials using the revPBE+D2 approach

\begin{tabular}{llllll}
\hline & & $a$ & $\mathrm{~B}$ & $\phi$ & $\Phi$ \\
\hline $\mathrm{Ru}$ & $\mathrm{q} 16[7 \mid 3221]$ & 2.685 & 323 & 0.225 & 5.01 \\
& q8 [6|212] & 2.718 & 320 & 0.218 & 5.00 \\
$\mathrm{Rh}$ & q17 [6|3221] & 3.796 & 250 & 0.178 & 5.13 \\
& q9 [6|2221] & 3.813 & 250 & 0.172 & 5.14 \\
& q9 [6|212] & 3.836 & 234 & 0.167 & 5.20 \\
\hline
\end{tabular}

Lattice constant $a[\AA]$, bulk modulus B [GPa], surface energy $\phi[\mathrm{eV} /$ $\left.\AA^{2}\right]$, and work function $\Phi[\mathrm{eV}]$. The lattice constant of Ru is calculated at a fixed $c / a$ value

the calculations of the $h$-BN/TM interfaces, with significant improvements in the computational performance.

\subsection{Hexagonal boron nitride}

To test the basis sets and pseudopotentials for the elements $\mathrm{B}$ and $\mathrm{N}$, we calculated properties of bulk $h$-BN. These systems also provide insight into the performance of the empirical vdW correction to the density functional for weak interactions. Results are shown in Table 4 for $h$-BN with $\mathrm{AA}^{\prime}$ and $\mathrm{AB}$ stacking. The $\mathrm{AA}^{\prime}$ stacking contains alternating $\mathrm{N}$ and $\mathrm{B}$ atoms along the direction perpendicular to the $\mathrm{BN}$ hexagonal plane. In the $\mathrm{AB}$ stacking, the second layer is shifted by a third of the unit cell along the in-plane (11) direction.

The dispersion correction is essential to obtain good agreement with experiment for the interlayer distance because the GGA functionals do not describe the vdW interactions. In particular, both variations of the Grimme potential (D2 and D3) give a slightly too short interlayer separation between the hexagonal $\mathrm{BN}$ planes, while the calculated bond lengths are not affected by the dispersion correction and agree well with experiment and previous theoretical estimates.

\section{$4 h$-BN/metal interface}

Thermal decomposition of borazine on hot TM surfaces can lead to the formation of a Moiré lattice of a highly regular and corrugated $h$-BN monolayer on the hexagonal metallic surface (for the fcc metals (111) and for Ru the $\mathrm{hcp}(0001)$ orientation). The periodicity of such superstructures at the $h$-BN/metal interface depends strongly on the lattice constant mismatch between $h$-BN and metal, but can also be influenced by the preparation details [63]. We investigate four systems: two where the lattice constant mismatch is almost $10 \%$ and the $h$-BN overlayer is strongly corrugated $(\mathrm{Rh}, \mathrm{Ru})$, forming the so-called nanomesh [1], and two with small mismatch and practically no corrugation $(\mathrm{Ni}, \mathrm{Cu})$. These systems have previously been investigated using different simulation methods and models. Structures and bonding of $h-\mathrm{BN} / \mathrm{Rh}(111)$ and $h$-BN/ $\mathrm{Ru}(0001)$ were studied in detail by applying LAPW models $[22,24]$. The $h$-BN/Ni(111) system has attracted attention due to the surface magnetization [20,21, 25, 26], which is retained after $h$-BN growth. Results on the $h-\mathrm{BN} / \mathrm{Cu}(111)$ systems have been reported within an extended study on several TMs [21]. More theoretical work on these systems is available in the context of additional molecular interactions (water on $h$-BN/Rh(111) [64, 65], metal atoms on $h$-BN/Rh(111) [27, 66], hydrogen adsorption and intercalation $[18,67])$, and in direct connection with experiments $[2,5,10,19,23,68]$.

The mismatch between $h$ - $\mathrm{BN}$ and metal is a fundamental parameter that determines binding and corrugation of the $h$-BN monolayer. For this reason, we choose the size of the simulation cell that reproduces exactly the experimental mismatch using as reference value the equilibrium lattice constant of $h$-BN as obtained with our setup, i.e., $2.517 \AA$. This results in an effective lattice constant $a$ of $3.801 \AA$ for $\mathrm{Rh}, 3.628 \AA$ for $\mathrm{Cu}, 3.543 \AA$ for $\mathrm{Ni}$, and $2.724 \AA$ for $\mathrm{Ru}$. Structural optimizations are typically started from a flat
Table 4 Crystal properties of the bulk $h$-BN at two different stackings, $\mathrm{AA}^{\prime}$ and $\mathrm{AB}$, with either no dispersion correction or DFT-D2 [31] and DFT-D3 [32] formalisms

The polymorphs are energetically degenerate within $1 \mathrm{meV}$. Cell parameter $a$, interlayer distance $d_{\text {layers }}$, and B-N bond distance $d_{\mathrm{B}-\mathrm{N}}[\AA]$. Other DFT calculations (with PW formalism with the LDA $\mathrm{XC}$ functional) and experimental results are shown for comparison

\begin{tabular}{|c|c|c|c|c|c|c|c|}
\hline & \multicolumn{3}{|c|}{ GPW: revPBE } & \multicolumn{2}{|c|}{ PAW: revPBE } & \multirow{2}{*}{$\begin{array}{l}\text { PW-LDA } \\
\text { [59] }\end{array}$} & \multirow[t]{2}{*}{ Experiments } \\
\hline & DFT & DFT-D2 & DFT-D3 & DFT & DFT-D2 & & \\
\hline \multicolumn{8}{|c|}{$h$-BN: $\left(A A^{\prime}\right)$} \\
\hline$a$ & 2.516 & 2.512 & 2.511 & 2.521 & 2.515 & 2.486 & $2.50[60]$ \\
\hline$d_{\text {layers }}$ & 4.145 & 3.154 & 3.224 & 5.262 & 3.076 & 3.219 & $3.33[60]$ \\
\hline \multirow[t]{3}{*}{$d_{B-N}$} & 1.453 & 1.450 & 1.450 & 1.455 & 1.452 & 1.435 & $1.44[61]$ \\
\hline & \multicolumn{3}{|c|}{ GPW: revPBE } & \multicolumn{2}{|c|}{ PAW: revPBE } & PW-LDA & Experiments \\
\hline & DFT & DFT-D2 & DFT-D3 & DFT & DFT-D2 & {$[62]$} & \\
\hline \multicolumn{8}{|c|}{$h$-BN: $(A B)$} \\
\hline$a$ & 2.516 & 2.511 & 2.510 & 2.521 & 2.514 & 2.478 & \\
\hline$d_{\text {layers }}$ & 4.148 & 3.135 & 3.199 & 5.318 & 3.041 & 3.279 & \\
\hline$d_{\mathrm{B}-\mathrm{N}}$ & 1.453 & 1.450 & 1.449 & 1.455 & 1.451 & 1.431 & \\
\hline
\end{tabular}


$h$-BN terminating a slab of four or seven metallic layers and are stopped when the largest gradient is smaller than $4 \times 10^{-4}$ Hartree/Bohr. The revPBE-GGA is used as the exchange-correlation functional and is augmented with the empirical DFT-D3 dispersion correction unless otherwise stated.

\section{1 $h$-BN adsorbed on $\mathrm{Rh}(111)$}

Full models of the $h-\mathrm{BN} / \mathrm{Rh}(111)$ unit cell have been studied previously $[22,24,27]$. In this section, we will extend our results [64] and compare them to other studies.

The lattice mismatch between $h$ - $\mathrm{BN}$ and $\mathrm{Rh}(111)$ is quite large, the (111) lattice constant of Rh being $-7.0 \%$ larger than that of $h$-BN. Such a mismatch does not allow a commensurate growth of the overlayer. Instead, a superstructure constituted of $13 \times 13 h$-BN units on a $12 \times 12$ $\mathrm{Rh}$ (111) surface has been observed. In principle, only a slight distortion of the $\mathrm{B}-\mathrm{N}$ bond length would be required to match with a flat $13 \times 13 h$-BN layer on top of the $12 \times 12 \mathrm{Rh}(111)$ units. This is an overall contraction of the bonds by $1.4 \%$ from 1.45 to $1.43 \AA$ and corresponds to an energy increase of $0.02 \mathrm{eV}$ per BN pair. This has to be compared with a stretching energy of $0.40 \mathrm{eV} / \mathrm{BN}$ for the $1 \times 1$ commensurate structure. In fact, the adsorbed $h$-BN layer is corrugated, in order to optimize the BN-Rh binding over a larger area. The extent of this area depends on the registry of $h$-BN over the $\mathrm{Rh}(111)$ surface.

\subsubsection{Quasi-commensurate adsorption of $h-B N$ on Rh(111)}

To gain a deeper insight into how the registry affects the binding properties, we first investigate models of smaller size, where the one to one commensurate structure is obtained by stretching the $h$-BN overlayer. In these models, we use a $\mathrm{Rh}(111)$ slab of seven layers and $6 \times 6$ lateral unit cells. The slab with the q17 pseudopotential for $\mathrm{Rh}$ is terminated on both sides with the stretched $6 \times 6 h$-BN layer to match the lattice constant $3.801 \AA$ of Rh. The $h$-BN layer can be arranged with nitrogen on top of a $\mathrm{Rh}$ atom and $\mathrm{B}$ on the next hollow site, namely the (N-top,B-fcc) and (N-top,B-hcp) registries, or with nitrogen occupying a hollow site, giving rise to the possible ( $\mathrm{N}-\mathrm{fcc}, \mathrm{B}-\mathrm{top}$ ), (N-hcp,B-top), (N-fcc,B-hcp), or (N-hcp,B-fcc) registries. These test systems are well suited to investigate the importance of the dispersion energy since they act as models for the different bonding situations in the full system. The registries with $\mathrm{N}$ atop are the most stable structures, whereas when $\mathrm{N}$ occupies a hollow site, the binding is weaker and lateral movement has to be constrained in geometry optimizations to keep the overlayer in such a position. In Table 5, interaction energy, work function, as well as some structural properties are
Table 5 Properties of the quasi-commensurate $h$-BN/Rh(111) system, using the symmetric slab model at the four registries: Interaction energies [eV/BN], work function $\Phi[\mathrm{eV}]$, and distances and buckling $[\AA]$

\begin{tabular}{llllll}
\hline $\mathrm{N}$ & $\mathrm{B}$ & $E_{\text {int }}$ & $\Phi$ & $d_{\min }(\mathrm{N})$ & $b_{\mathrm{B}-\mathrm{N}}$ \\
\hline top & fcc & -0.621 & 3.07 & 2.184 & 0.139 \\
top & hcp & -0.587 & 3.07 & 2.193 & 0.141 \\
fcc & top & -0.323 & 3.83 & 2.590 & 0.088 \\
hcp & top & -0.325 & 3.76 & 2.608 & 0.085 \\
\hline
\end{tabular}

summarized for the four investigated commensurate $1 \times 1$ models.

The stretched overlayer remains overall flat in all the registries, even though the $\mathrm{B}-\mathrm{N}$ bond buckles. The most stable structure is the (N-top,B-fcc) registry, with a minimum N-Rh distance of about $2.18 \AA$. The (N-top,B-hcp) is very similar, only $0.034 \mathrm{eV} / \mathrm{BN}$ less stable than the (N-top,B-fcc) registry. Since the energy cost for a 1-to-1 matching of $h$ - $\mathrm{BN}$ on $\mathrm{Rh}(111)$ is quite large, the adsorption energy is only about $-0.14 \mathrm{eV}$ per BN pair in the $\mathrm{N}$-top structures, while it is positive in all the N-hollow models. In the latter case, the weak binding is not sufficient to compensate the energy cost for the distortion of the $h$-BN layer. When $\mathrm{N}$ occupies hollow sites, the vertical distance from $\mathrm{Rh}(111)$ increases and the buckling is reduced. The buckling can be explained considering the weakened $\mathrm{B}-\mathrm{N}$ bond, due to the stretching of the $h$-BN layer and a partial charge redistribution. Indeed, due to the presence of the metal, the electronic charge localized around the $\mathrm{N}$ atoms is polarized towards the $\mathrm{Rh}$ slab. Hence, the positively charged $\mathrm{B}$ atoms get attracted towards the negatively charged interlayer region. This effect is more evident in the $\mathrm{N}$-top registry, where the $\mathrm{N}-\mathrm{Rh}$ interactions and the polarization of $\mathrm{N}-p_{z}$ electrons are stronger. In this case, we can consider the $\mathrm{N}-p$ band as hybridized with the metal $d$ band.

In order to characterize the electronic structure, we make use of the MLWFs. The displacements of the centres of charge, or Wannier centres (WC) of the MLWFs, with respect to the nuclear cores in different chemical environments can be used as an indicator of polarization. In free-standing $h$-BN, three such WCs are found along the three in-plane B-N bonds of each nitrogen atom. The fourth centre, representing the nitrogen lone pair, is located at the $\mathrm{N}$ atom. In the N-top structure, instead, the lone-pair WC is displaced into the interlayer region, indicating a polarization of the electronic charge distribution due to the interaction with the metal. As a consequence, the work function of the system is reduced from $5.01 \mathrm{eV}$ of bare $\mathrm{Rh}(111)$ to $3.07 \mathrm{eV}$. Such N-Rh binding interaction is not observed in the N-hollow configurations, and the lone-pair WC is located within the $h$-BN plane like in the 
free-standing $h$-BN. The work function in this case is $3.8 \mathrm{eV}$, almost independent of the specific registry.

In the (N-top,B-fcc) and (B-top, N-fcc) models, we have investigated the effect of the empirical dispersion correction term and the basis set superposition error (BSSE). The counterpoise correction method results in a $3 \%$ correction, or destabilisation, of the interaction energy in both cases. The interaction energy computed in the two models as function of the distance of the $h$-BN layer from the surface is displayed in Fig. 1. In these calculations, the structure has not been relaxed. In the case of strong interaction (N-top), DFT(revPBE) alone results in bonding at distances between 2.1 and $2.5 \AA$, with a minimum at about $2.3 \AA$. This bonding is slightly enhanced by the dispersion correction, which results in a decrease in the equilibrium distance. In contrast, the weakly bound system has all its binding energy from the dispersion contribution. The effect of the actual type of dispersion correction used (D2 vs. D3) is minor, the D3 correction always being slightly weaker than the D2 correction. In the full nanomesh, the dispersion forces play an important role in the weakly bound region (wire). The effect is that the wire lies closer to the metal surface, thus reducing the overall corrugation. The same effect has been seen in graphene systems [69].

\subsection{2 h-BN/Rh(111) nanomesh}

The full supercell for the nanomesh is constructed with the metallic slab of $12 \times 12$ lateral unit cells of $\mathrm{Rh}(111)$ terminated with a $13 \times 13 h$-BN overlayer. We tested several different models by changing the thickness of the slab, from four to seven layers, and pseudopotential and basis set of $\mathrm{Rh}$, i.e., both q17 and q9 pseudopotentials with the

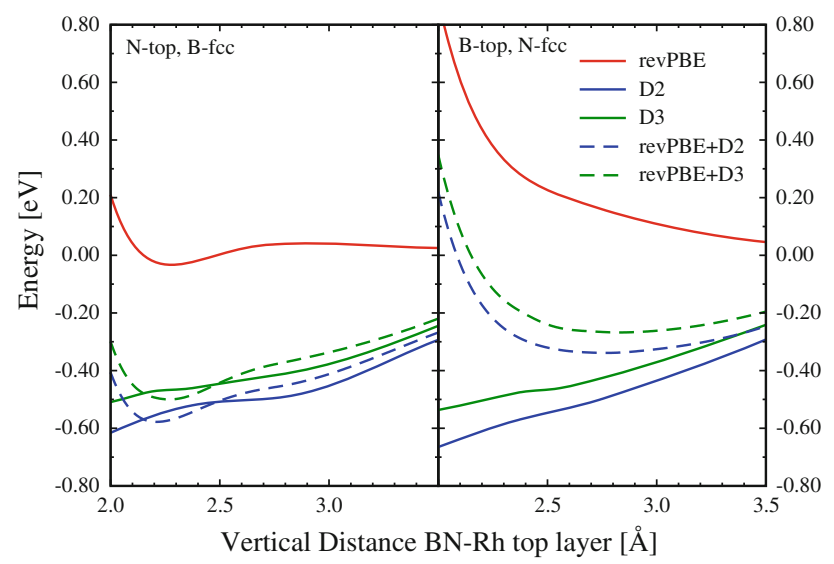

Fig. 1 Interaction energy as a function of the vertical displacement of the $h$-BN overlayer calculated in the $h$-BN/Rh(111) system: DFT(revPBE) contribution (red), and the contribution of only the dispersion D2 (blue) and D3 (green). The total potential energy can be obtained by summing red and blue (revPBE+D2, blue dashed) or red and green (revPBE $+\mathrm{D} 3$, green dashed) lines
[6|3221] and [6|212] basis sets, respectively. For B and N, we always use q3 and q5 pseudopotentials and [4/221] and [5|221] basis sets. The largest tested model is a symmetric slab of 7 layers (1008 Rh atoms) and one $h$-BN overlayer per side (338 BN pairs), for a total of 1,684 atoms. With the q17 pseudopotential and the largest [613221] basis set for $\mathrm{Rh}$, this amounts to 19,840 electrons and 34,996 basis functions. Nevertheless, similar structural and electronic properties of the nanomesh are obtained with the computationally more convenient setup employing q9 pseudopotential and [6/212] basis set. In what follows, we report the results for the seven layer slab with $h$-BN on one side only (non-symmetric system) and the bottom-most layer is kept fixed with the atoms in bulk positions. With this model, we allow the relaxation of the metal layers deeper inside the slab. This is important in order to be able to estimate how much the interaction with $h$-BN affects the substrate.

Upon starting the optimization of the full system with a flat and contracted $h$-BN overlayer ( $1.4 \%$ contraction of B-N bond length), it slowly adjusts to the substrate, building up a corrugation pattern and modulation of the B-N bond length. From the height colour map in Fig. 2a), where the $\mathrm{B}$ and $\mathrm{N}$ atoms are coloured in red (pore) and blue (wire), two flat regions are clearly identified. The pore region contains the atoms that are closer to the metal (2.2-2.4 $\AA$ ). Here, the registry is approximately (N-top,Bfcc) and the B-N bond length tends to be stretched with respect to free-standing $h$-BN (see Fig.2b), in order to maximize the $\mathrm{N}-\mathrm{Rh}$ contact and thus the pore area. For the same reason, $\mathrm{Rh}$ atoms below the pore have a slightly shorter Rh-Rh bond distance of $2.67 \AA$. The shape of the pore is approximately hexagonal and it is centred on three on top $\mathrm{N}$ atoms. The wire region lies about $1 \AA$ higher, between 3.2 and $3.4 \AA$. Here, the $\mathrm{B}$ atoms are approximately on top and the bond length is contracted. The steep change in height involves only two BN rows (yellow and green in the figure). The pore region corresponds to about $20 \%$ of the overlayer. The wire involves slightly more than $50 \%$ of the area. The adsorption energy $E_{\text {ads }}$ per BN pair is calculated to be $-0.25 \mathrm{eV}$. This value contains the energy loss due to the contraction of the flat $h$-BN layer, $0.02 \mathrm{eV} / \mathrm{BN}$, the energy loss due to the corrugation of the contracted overlayer, $0.03 \mathrm{eV} / \mathrm{BN}$, and the energy loss due to the corrugation of the $\mathrm{Rh}$ layers, induced by the interaction with $h$-BN. The bare interaction energy between $h$-BN and metal amounts to $-0.31 \mathrm{eV} / \mathrm{BN}$.

The hybridization of the $\mathrm{N}$ lone pairs with the $\mathrm{Rh} d$ band is responsible for the bonding interaction in the pore region. The charge density difference map reported in Fig. 3 shows that modifications of the charge distribution due to the interaction between $h$-BN and $\mathrm{Rh}$ are visible only in the region of the pore. In the region between the metal surface and $h$ - $\mathrm{BN}$, accumulation of charge is 
Fig. 2 a Height colour map of the $\mathrm{B}$ and $\mathrm{N}$ atoms, measured relative to the average height of the topmost Rh layer, in the $h$ $\mathrm{BN} / \mathrm{Rh}(111)$ nanomesh. The height ranges for each colour are given in the legend of the figure. The small grey spheres represent the $\mathrm{Rh}$ atoms of the slab underneath. b B-N bond length versus the height of the $\mathrm{N}$ atoms over the topmost $\mathrm{Rh}$ layer. The green and red horizontal lines indicate the equilibrium $\mathrm{B}-\mathrm{N}$ bond length and the bond length of flat $13 \times 13 h$-BN over the $12 \times 12$ $\mathrm{Rh}(111)$ units, respectively

Fig. 3 a Top view of the charge density difference in the $h$-BN/ $\mathrm{Rh}(111)$ nanomesh. The red and the black iso-surfaces in the top view indicate a difference of +0.005 and of $-0.005 \mathrm{e} / \AA^{3}$, respectively. b, c Slice cutting through the middle of the pore through $\mathrm{N}$ and $\mathrm{B}$ atoms, respectively. The range of the colour map from blue to red corresponds to values from -0.005 to +0.005 e $/ \AA^{3}$
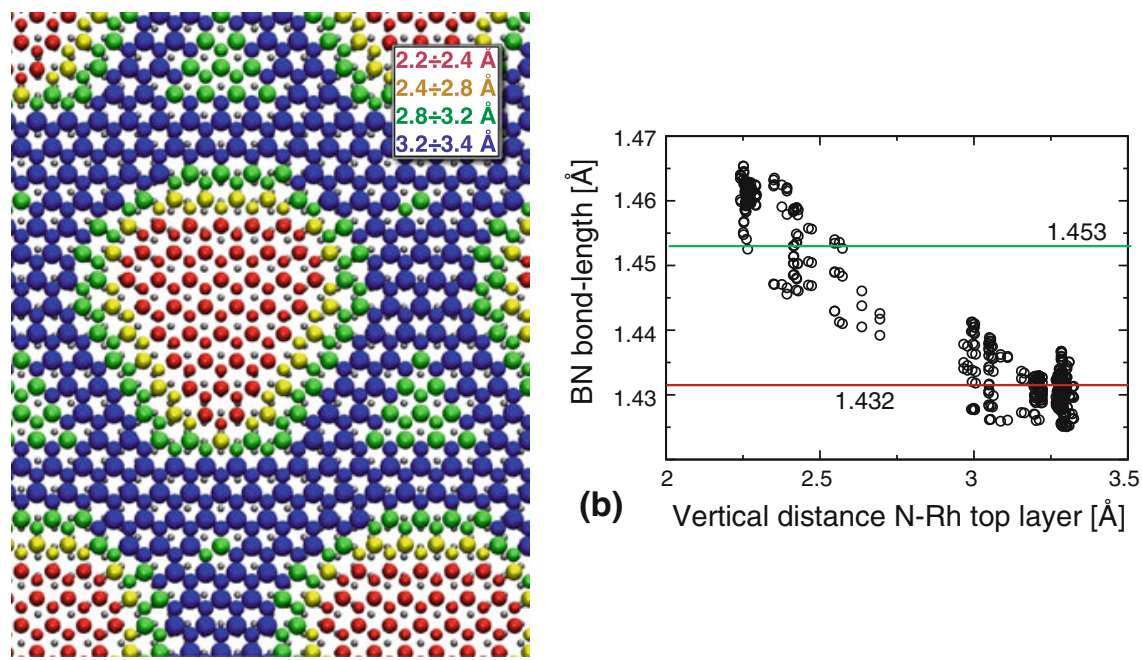

(a)

(b)

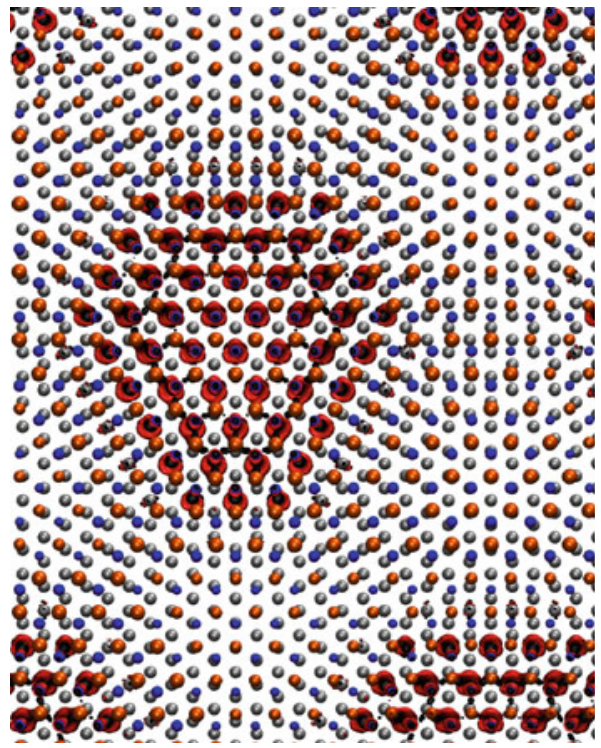

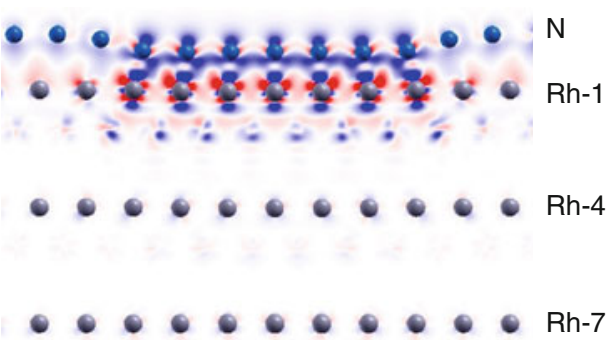

(b)

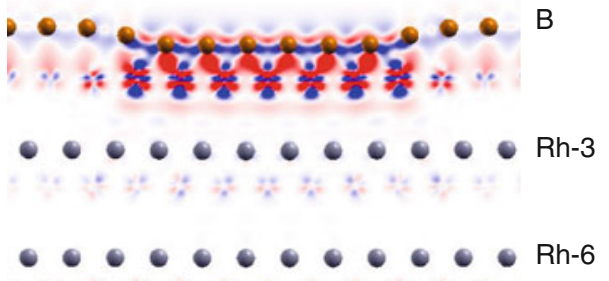

(c) observed at the first Rh layer (red iso-surface at $+0.005 \mathrm{e} / \AA^{3}$ and red areas in the volume slice). The displaced charge comes mainly from the $\mathrm{N}$ atoms and from the first layer of $\mathrm{Rh}$ atoms, where depletion areas (black iso-surface) are visible. The blue areas in the volume slice in Fig. $3 b$ indicate that charge is subtracted from the area around the $\mathrm{N}$, whereas red areas are in the interlayer region under both $\mathrm{N}$ and $\mathrm{B}$. All other parts of the metal as well as the wire region of the nanomesh do not show any charge redistribution. The description of the charge distribution via Mulliken atomic charges shows that $\mathrm{N}$ atoms turn out to be less negative than in the free-standing $h$-BN, while $\mathrm{B}$ atoms tend to keep their positive charge. Overall, the $h$-BN layer is partially positive and the missing negative charge is located at the slab surface, below the pore.

In Fig. 4a, the simulated STM topography, obtained at a bias potential of $-1 \mathrm{eV}$ with the method described in Sect. 2 , is reported. The iso-current surface has been generated starting with a value of isodensity $n_{b}=10^{-6} \mathrm{e} / \AA^{3}$; it lies about $3 \AA$ above the $h$-BN overlayer. The overall corrugation of this topography map is about $1 \AA$, as also shown by the height-profile obtained along the black-dashed line crossing through the centre of the pore region. The vertical position of the WCs can be used to show the correlation of amount of bonding with the distance of the overlayer from the metal surface. In Fig. $4 \mathrm{~b}$, the distance along the $\mathrm{z}$ axis of each lone-pair WC from the closest $\mathrm{N}$ atom is reported on the ordinate, while on the abscissa is the height of the corresponding $\mathrm{N}$ atom above the $\mathrm{Rh}$ surface. In this plot, the height-to-polarization correlation is clearly visible. In particular, we notice that in the presence of the $\mathrm{Rh}(111)$ substrate, the WC-N distance increases when the $\mathrm{N}$ is closer to the metal (red squares). This effect is not visible in the corrugated free-standing $h$-BN (green squares), where the lone-pair WCs remain at about $0.2 \AA$ from N, irrespective of the height of $\mathrm{N}$. The charge density 

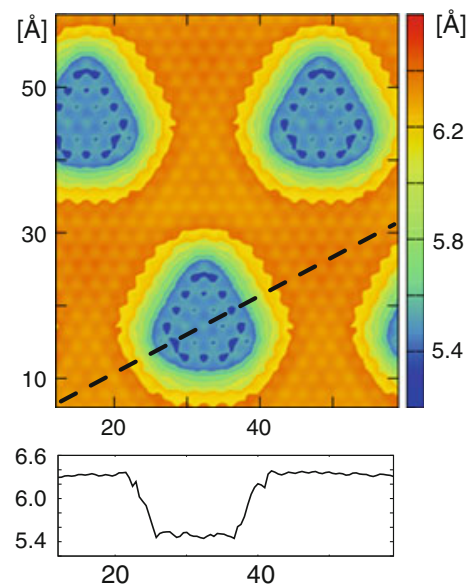

(a) $\quad x$ axis $[\AA]$

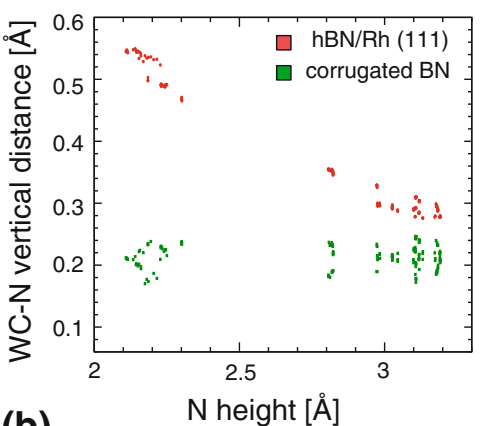

(b)

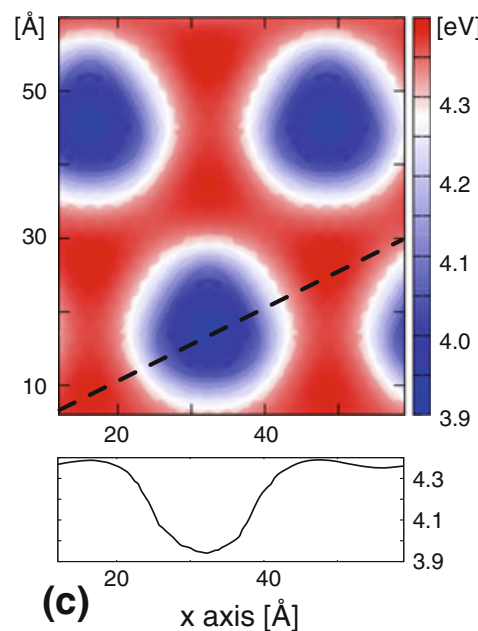

Fig. 4 a Simulated STM Tersoff-Hamann iso-current surface obtained at a bias potential of $-1 \mathrm{eV}$ at the $h$-BN/Rh(111) nanomesh. The axis units are $\AA$. b Absolute vertical distance of the lone-pair WC from $\mathrm{N}$ versus height of $\mathrm{N}$ at the nanomesh (red) and at the corrugated

polarization in the pore region directly leads to a strong modulation of the electrostatic potential and, consequently, of the local work function. Figure $4 \mathrm{c}$ contains the map of the local work function over the simulated STM iso-current surface displayed in panel a). The resulting work function map can be compared to experimental $\mathrm{dI} / \mathrm{dz}$ maps [68]. Along the line crossing diagonally through the pore, we estimate a difference in work function between wire and pore of about $0.45 \mathrm{eV}$. It is this strong change in electrostatic potential that is responsible for the trapping of molecules and atoms in the pore [64].

\section{$4.2 h$-BN adsorbed on $\mathrm{Ru}(0001)$}

The $a$ parameter of the Ru hexagonal lattice is $8.2 \%$ larger than that of $h$-BN. Hence, it is reasonable to expect that similar structures as those observed on $\mathrm{Rh}(111)$ can be formed on the $\mathrm{Ru}(0001)$ surface. Indeed, the formation of a nanomesh of $h$-BN on $\mathrm{Ru}(0001)$ was reported first in 2007 [3]. The periodicity of 13-on-12 was considered to be the most likely [70] and was therefore subsequently used in the theoretical studies by Laskowski and Blaha [24] and Wang and Bocquet [27]. Comparison with experiments also supported this periodicity, as $h$-BN deposited on thin $\mathrm{Rh}(111)$-films grown on yttrium-stabilized zirconia on $\mathrm{Si}(111)$ [63] resulted in a 14-on-13 structure: It was argued that the slightly smaller lattice constant of the Rh-film compared to bulk $\mathrm{Rh}(111)$ and the slightly different thermal expansion coefficients are responsible for the formation of this larger superstructure. Extrapolating this line of argument to a $\mathrm{Ru}(0001)$ single crystal, it was predicted that either a 12 -on-11 or a 13-on-12 nanomesh superstructure would be formed at the growth temperature of $900 \mathrm{~K}$.

but free-standing $h$-BN with the same coordinates as in the full system (green). c Modulation of the local work function calculated at the Tersoff-Hamann iso-current surface. The values of the work function are in $\mathrm{eV}$

However, using surface X-ray diffraction [71], a 14-on-13 structure was found. According to the proposed interpretation, energy minimization from the stronger bonding of $h$-BN to $\mathrm{Ru}$ in comparison with $h$ - $\mathrm{BN} / \mathrm{Rh}(111)$ overcomes the increased strain energy and leads to the formation of a larger superstructure, 14-on-13, rather than the smaller 13-on-12.

Here, we consider both the proposed superstructures for $h$-BN on $\mathrm{Ru}(0001)$, i.e., the 13 -on- 12 and the 14 -on- 13 , and compare to the 13 -on-12 $h$-BN on $\mathrm{Rh}(111)$ nanomesh. In these calculations, we employ the q8 pseudopotential and the [6|212] basis set for Ru. The models contain seven $\mathrm{Ru}$ layers terminated by the $h$-BN overlayer on one side only. On the opposite side of the slab, the Ru atoms of the terminating layer are fixed at bulk positions. The flat $13 \times 13$ $h$-BN overlayer can match the $12 \times 12 \mathrm{Ru}(0001)$ surface without distortion, whereas in the 14-on-13 superstructure, the B-N bond length has to be elongated by $0.5 \%$. However, as observed already on $\mathrm{Rh}(111)$, starting the optimization from a flat overlayer, a strongly corrugated structure is formed in both registries, where lower and higher regions correspond to elongated and contracted $\mathrm{B}-\mathrm{N}$ bonds, respectively. The 14-on-13 superstructure turns out to be energetically favoured, with an adsorption energy $0.012 \mathrm{eV} / \mathrm{BN}$ lower than that of the 13-on-12. The reason is the larger low and flat region (pore) with approximately (N-top,B-fcc) registry, where the $\mathrm{N}-\mathrm{Ru}$ interaction is maximized. On $\mathrm{Ru}$, the corrugation is more pronounced than on $\mathrm{Rh}$, being about $1.7 \AA$ in 13 -on- 12 and $1.5 \AA$ in 14-on-13 models. The height colour map of the 14-on-13 structure is reported in Fig. 5a. The area of the wire region (blue) is substantially smaller compared to the nanomesh on $\mathrm{Rh}$, indicating a better contact between the overlayer 
Fig. 5 a Height colour map of the $\mathrm{B}$ and $\mathrm{N}$ atoms, as measured relative to the average height of the topmost $\mathrm{Ru}$ layer in the optimized 14-on-13 $h$-BN/Ru structure. The height ranges for each colour are given in the legend. The small grey spheres represent the $\mathrm{Ru}$ atoms of the slab underneath. b B-N bond length versus the height of the $\mathrm{N}$ atoms above the topmost $\mathrm{Ru}$ layer. The green and red horizontal lines indicate the equilibrium $\mathrm{B}-\mathrm{N}$ bond length and the bond length of flat $14 \times 14 h$-BN over the $13 \times 13$ $\mathrm{Ru}(0001)$ units

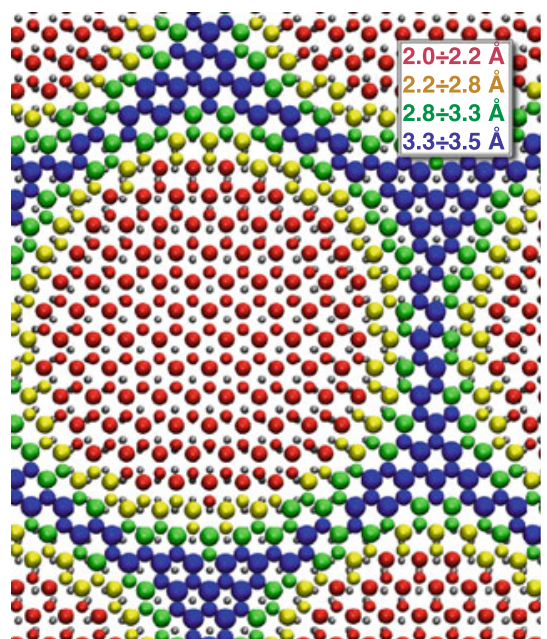

(a) $10.0 \%$

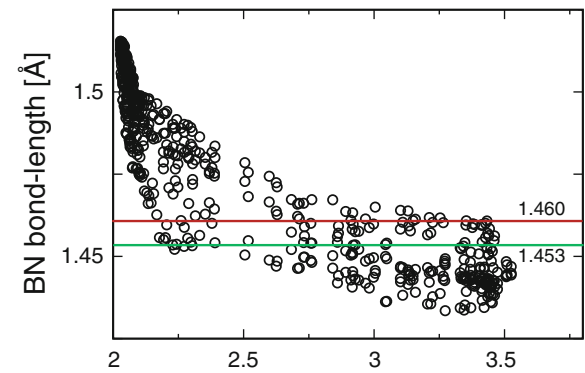

(b) Vertical distance N-Ru top layer $[\AA]$ and the $\mathrm{TM}$ in the present case. In the pore, the $\mathrm{B}-\mathrm{N}$ bond length is elongated in order to optimize the (N-top,B-fcc) registry, with bonds as long as $1.52 \AA$. The overlayer keeps a good contact with the substrate even at the outer ring of the pore region, where the $\mathrm{N}$ atoms are displaced from the top sites. Hence, the interaction area on $\mathrm{Ru}(0001)$ is more extended, including also the region of the overlayer where the $\mathrm{B}-\mathrm{N}$ bond is located above $\mathrm{Ru}$, with approximately bridge-type registry. This is clearly visible in the charge density difference map reported in Fig. 6. The charge redistribution affects more than $50 \%$ of the overlayer. Electronic charge is displaced from the $\mathrm{N}$ lone pairs and the $\mathrm{B}-\mathrm{N}$ bonds (black iso-surface) towards the metal, thus creating an accumulation of charge in the interlayer region (red iso-surface). Moreover, the footprint of the $h$-BN-Ru interaction extends deeper into the metallic slab than what is observed in the $\mathrm{Rh}(111)$ system, namely the plane cutting through the pore (panels $\mathrm{b}$ and $\mathrm{c}$ in Fig. 6) shows significant charge redistribution between the second and third Ru layers. Also the corrugation of the metallic layers below the nanomesh is larger than in Rh (cf. data in Table 6). Due to the more effective interaction, even if the stronger corrugation causes larger energy loss, i.e., $0.14 \mathrm{eV} / \mathrm{BN}$ in the 14-on-13, the adsorption energy on $\operatorname{Ru}(0001)$ is more than $0.1 \mathrm{eV}$ per BN pair lower than on $\mathrm{Rh}(111)$.

The nature of the electronic interaction between $\mathrm{Ru}$ and the overlayer can be better understood from the PDOS. Figure 7 displays the PDOS on $\mathrm{N}$ and $\mathrm{B} p$-states, distinguishing between the contributions from the atoms belonging to the pore region and those in the wire region. The interaction with the metal mostly affects the occupied $p$-states on the pore $\mathrm{N}$. The corresponding band shifts to lower energy due to the hybridization with the $\mathrm{Ru} d$ band. On the contrary, the B PDOS show much smaller changes between pore and wire, suggesting that B is less directly involved in the orbital re-hybridization.
Fig. 6 a Top view of the charge density difference in the 14-on$13 h$-BN/Ru nanomesh. The red and the black iso-surfaces in the top view indicate a difference of +0.005 and of $-0.005 \mathrm{e} / \AA^{3}$, respectively. b, $\mathbf{c}$ Plane cutting in the centre of the pore through $\mathrm{N}$ and through $\mathrm{B}$, respectively. The range of the colour map from blue to red corresponds to values from -0.005 to +0.005 $\mathrm{e} / \AA^{3}$

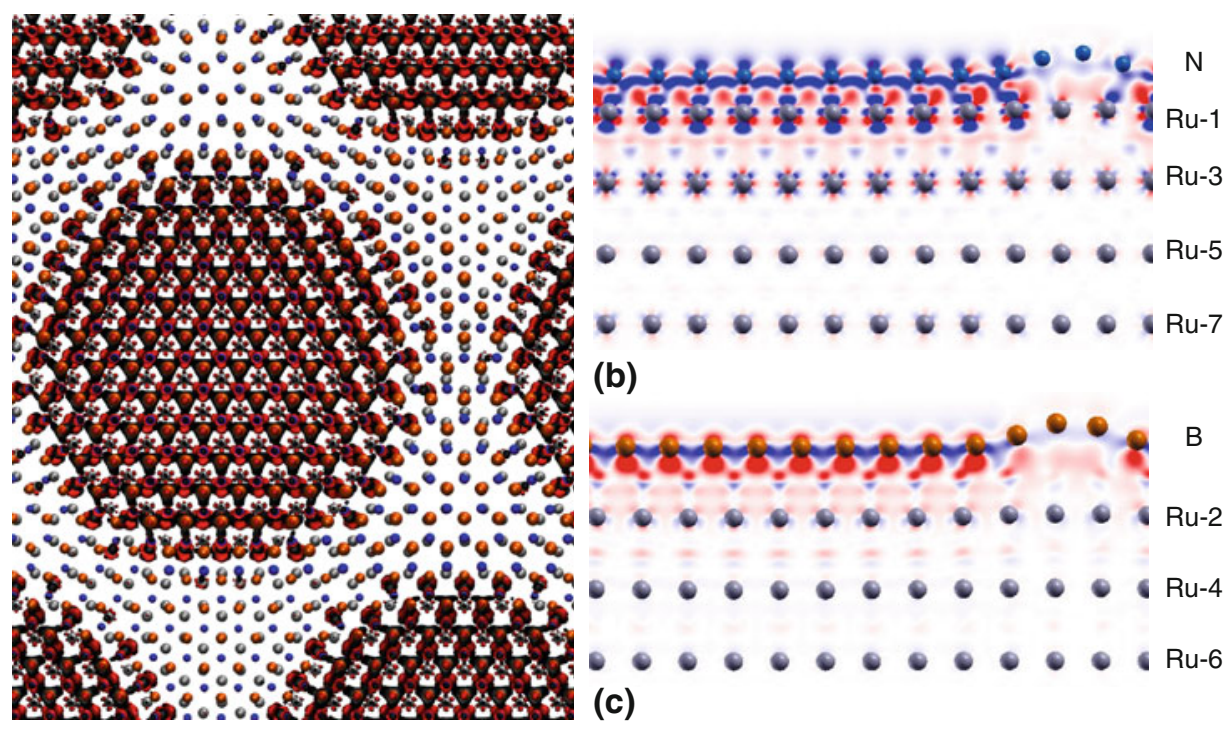


Table 6 Summary and comparison of results for $h$-BN on $\mathrm{Rh}(111)$ and $\mathrm{Ru}(0001)$

\begin{tabular}{lccc}
\hline & $\begin{array}{c}h \text {-BN/Rh } \\
\text { 13-on-12 }\end{array}$ & $\begin{array}{c}h \text {-BN/Ru } \\
13 \text {-on-12 }\end{array}$ & $\begin{array}{c}h \text {-BN/Ru } \\
14 \text {-on-13 }\end{array}$ \\
\hline$E_{\text {BNdist }}$ & 0.022 & 0.0001 & 0.004 \\
$E_{\text {ads }}$ & -0.254 & -0.367 & -0.380 \\
$E_{\text {int }}$ & -0.310 & -0.520 & -0.542 \\
$E_{\mathrm{BNcorr}}$ & 0.032 & 0.134 & 0.139 \\
$d_{\text {min }}(\mathrm{N})$ & 2.208 & 2.186 & 2.186 \\
$d_{\text {min }}(\mathrm{B})$ & 2.128 & 2.082 & 2.076 \\
$\Delta h_{\mathrm{N}}$ & 1.082 & 1.665 & 1.497 \\
$\Delta h_{\mathrm{B}}$ & 1.175 & 1.754 & 1.640 \\
$\Delta h_{1 \mathrm{stTM}}$ & 0.153 & 0.258 & 0.247 \\
$\Delta h_{\text {nndTM }}$ & 0.157 & 0.248 & 0.256 \\
$\Delta h_{\text {rdTM }}$ & 0.125 & 0.202 & 0.212 \\
$\Delta h_{\mathrm{STM}}$ & 1.0 & 1.7 & 1.6 \\
$\Delta \Phi$ & 0.5 & 0.36 & 0.35 \\
pore $\%$ & 20 & 43 & 46 \\
$(<2.4$ on $\mathrm{Rh} ;<2.2$ on Ru) & & & \\
wire $\%$ & 52 & 23 & 19 \\
$(>3.2$ on $\mathrm{Rh} ;>3.3$ on Ru $)$ & & & \\
\hline
\end{tabular}

Energies are given in eV/BN, distances and corrugations in $\AA$, work functions in $\mathrm{eV}$. $E_{\mathrm{BNdist}}$ is the distortion energy of the flat freestanding $h$-BN, $E_{\text {ads }}$ the adsorption energy, $E_{\text {int }}$ the interaction energy, and $E_{\mathrm{BNcorr}}$ the corrugation energy of the distorted free-standing $h$-BN. $d_{\min }(\mathrm{N}), d_{\min }(\mathrm{B})$, and $\Delta h_{\mathrm{B}, \mathrm{N}}$ are defined in the text. $\Delta h_{\mathrm{TM}}$ of the first three TM layers is computed as the difference between minimum and maximum height of the metal atoms within each corresponding layer. The corrugation of the simulated STM topography, $\Delta h_{\mathrm{STM}}$, is obtained from the iso-current surface at a bias of $-1 \mathrm{eV}$, and the modulation of the local work function $\Delta \Phi$ is computed at this same iso-current surface

\section{$4.3 h$-BN adsorbed on $\mathrm{Cu}(111)$}

Compared with other metal substrates, copper has so far received little attention for $h$-BN adsorption. An experimental study using XAS and photoemission spectroscopy [8] revealed only weak chemisorption of $h$ - $\mathrm{BN}$ on $\mathrm{Cu}(111)$ and a very flat layer. More recently, Laskowski et al. [21] included $\mathrm{Cu}$ in a systematic theoretical study of TM substrates for $h$-BN. They used the LAPW method to study 7-layer metal slabs with a commensurate $1 \times 1$ registry. They report results obtained with different $\mathrm{XC}$ functionals, LDA, PBE, and Wu-Cohen-GGA (WC-GGA) [72]. The LDA interaction energy is $-190 \mathrm{meV} / \mathrm{BN}$ for $h$-BN adsorbed in the (N-top, $\mathrm{B}-\mathrm{fcc}$ ) registry, the height over the $\mathrm{Cu}(111)$ surface $3.10 \AA$, and the B-N buckling $0.02 \AA$. On the other hand, $E_{\text {int }}$ is only $-10 \mathrm{meV} / \mathrm{BN}$ with the WCGGA functional and the monolayer turns out to be unbound (positive $E_{\text {int }}$ ) with $\mathrm{PBE}$. We note that no dispersion correction was applied in these calculations.
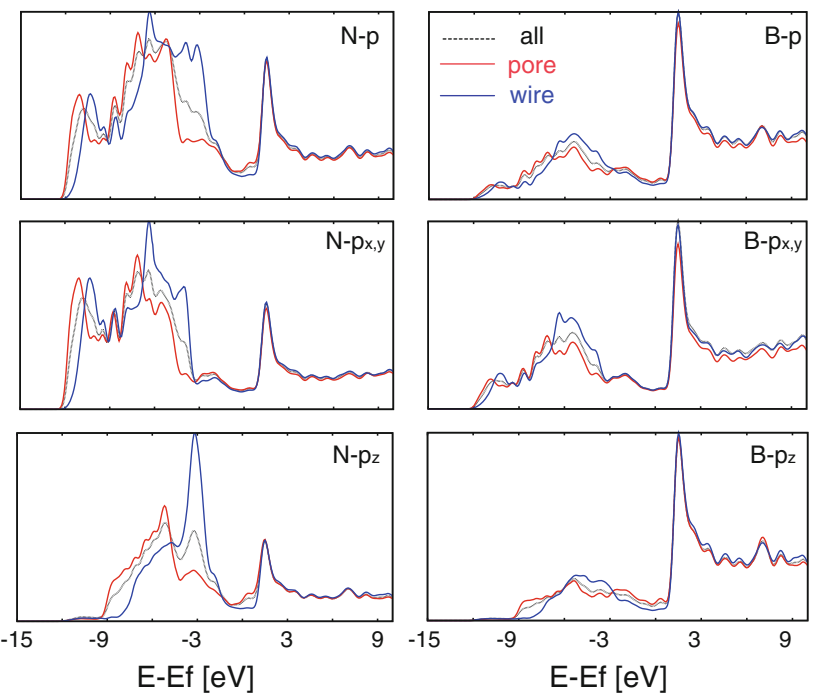

Fig. 7 PDOS in the $14-$ on- $13 h$-BN/Ru(0001) nanomesh on $\mathrm{N}$ and $\mathrm{B}$ $p$-states normalized by the number of atoms included in the projection. The PDOS obtained from all the $\mathrm{N}$ or $\mathrm{B}$ atoms in the overlayer, and only from the atoms in the pore and the wire are shown with black, red, and blue lines, respectively

In this work, the previously discussed setup is employed to determine adsorption energies and structural properties of the $h$-BN/Cu system with six different registries. The $\mathrm{Cu}(111)$ slab of seven $6 \times 6$ layers is symmetrically terminated on both sides by a $6 \times 6 h$-BN layer, located at an initial vertical distance of $2 \AA$. The $h$-BN and $\mathrm{Cu}$ lattices match quite closely, with $h$-BN having a $2.2 \%$ smaller inplane lattice parameter. In order to match the $\mathrm{Cu}(111)$ lattice, the $h$-BN layer is thus stretched, thereby elongating the bond lengths by the $2.2 \%$. Upon the optimization, the $\mathrm{B}-\mathrm{N}$ bond length is not exactly uniform over the layer anymore and these adjustments cause a minute offset with respect to the assigned registry and the observed small corrugation $(<0.03 \AA)$. Adsorption and interaction energies as well as some structural parameters from all the six structural optimizations are listed in Table 7.

As can be seen from both the adsorption energies and the $h$-BN-metal separation, the differences among the registries are quite small. $E_{\text {ads }}$ is always negative (i.e. binding) and very close to $E_{\text {int }}$, which is about $30-50 \mathrm{meV}$ smaller, except in (N-top,B-hcp) where the two energies are essentially equal. This is an indication that the monolayer remains largely unchanged upon adsorption, i.e., effective interaction is weak. The work function, $\Phi$, spans a range of $0.1 \mathrm{eV}$ from 3.62 to $3.71 \mathrm{eV}$. The systems are also similar in geometrical structure. In all registries, the adsorbate layer is approximately $3 \AA(2.99-3.03 \AA)$ from the metal surface and is very flat, as indicated by the small $h$-BN corrugation.

In contrast to previous studies, our work explicitly takes into account dispersion contributions to the adsorption 
Table 7 Properties of the commensurate $h$ - $\mathrm{BN} / \mathrm{Cu}(111)$ system, using the symmetric slab model in the six registries: adsorption and interaction energies $[\mathrm{eV} / \mathrm{BN}]$, work function $\Phi[\mathrm{eV}]$, and distances, corrugation and buckling $[\AA]$

\begin{tabular}{lllllllll}
\hline $\mathrm{N}$ & $\mathrm{B}$ & $E_{\text {ads }}$ & $E_{\text {int }}$ & $\Phi$ & $\begin{array}{l}d_{\min } \\
(\mathrm{B})\end{array}$ & $\begin{array}{l}d_{\min } \\
(\mathrm{N})\end{array}$ & $\Delta h_{\mathrm{N}}$ & $b_{\mathrm{BN}}$ \\
\hline top & fcc & -0.270 & -0.298 & 3.62 & 2.962 & 2.977 & 0.010 & 0.015 \\
top & hcp & -0.265 & -0.262 & 3.63 & 2.966 & 2.980 & 0.020 & 0.015 \\
fcc & top & -0.244 & -0.284 & 3.66 & 2.959 & 2.976 & 0.052 & 0.016 \\
hcp & top & -0.229 & -0.273 & 3.67 & 2.969 & 2.985 & 0.043 & 0.016 \\
fcc & hcp & -0.234 & -0.281 & 3.71 & 3.023 & 3.036 & 0.006 & 0.013 \\
hcp & fcc & -0.234 & -0.280 & 3.69 & 3.019 & 3.032 & 0.045 & 0.013 \\
\hline
\end{tabular}

energies. These appear to be significant in order to obtain a monolayer adsorbed in a stable way, as opposed to the very weakly bound or unbound systems. However, as vdW interactions are unspecific and only attractive, they do not make clear distinction among the various possible registries. Due to a small electronic effect, configurations with $\mathrm{N}$ atop turn out to be somewhat energetically favoured to the others, which confirms previous results [20, 21]. However, we cannot confidently distinguish between (N-top,B-fcc) and (N-top,B-hcp). The energetic differences between the remaining four registries are also too small to establish a well-defined hierarchy of stability.

We have investigated the influence of the $h-\mathrm{BN}-\mathrm{Cu}$ interaction on the electronic structure of the $h-\mathrm{BN}$ by comparing the PDOS and the charge density distribution. In general, the observed effects are small, and therefore, we do not show plots of the results here. By mapping the total density differences, only a slight polarization of the monolayer upon adsorption is revealed, largely independent of the registry. The major difference in the PDOS on the $p_{z}$ states of $\mathrm{N}$ between the (N-top,B-fcc) and the (N-fcc,B-hcp) configurations, the structures with the smallest and largest values of the work function, is a shift of the valence band by about $0.3 \mathrm{eV}$.

More recently, it has been shown [73] that the $-2.2 \%$ lattice mismatch between $\mathrm{Cu}(111)$ and $h$-BN causes the formation of Moiré patterns also at this interface, accompanied by small rotations of the overlayer with rotation angles from 1 to 10 degrees. The resulting superstructures have much larger unit cells and the simulations become much more challenging. Further discussion of this type of $h$ - $\mathrm{BN} / \mathrm{Cu}(111)$ superstructure, supported by both experiments and calculations, is reported in a separate work [73].

\section{$4.4 h$-BN adsorbed on $\mathrm{Ni}(111)$}

Several theoretical $[20,21,23,25,74]$ and experimental $[8$, $10,26]$ studies on the adsorption of $h$-BN on nickel surfaces have been published, focusing on various structural, energetic, and spectroscopic aspects. Among the possible metal substrates, the $\mathrm{Ni}(111)$ in-plane lattice constant $(2.49 \AA)$ matches that of $h$-BN most closely, allowing the formation of an almost perfectly commensurate overlayer. Since the earliest experiments of $h$-BN/Ni $[75,77]$, the nature of the bonding of the monolayer to the surface has been discussed repeatedly. Some authors have argued for a weak physisorption-like interaction [8], while others have proposed a strong(er), chemisorption-like binding [10].

Table 8 summarizes a number of previously published studies of this system, both theoretical and experimental. In particular, we list energetic (adsorption energy) and structural (interlayer distances and corrugations) features as well as the change in surface magnetic moment upon monolayer adsorption. A number of different adsorption geometries were considered by varying the registry of $h$-BN on $\mathrm{Ni}(111)$, leading in some cases to a notable variation in the obtained results. In general, however, all studies find the most stable adsorption registries to be those with $\mathrm{N}$ atop the $\mathrm{Ni}$ atoms and $\mathrm{B}$ either in the hcp or fcc sites, the latter typically slightly preferred. Furthermore, the $h$-BN layer tends to be slightly buckled in a way that places the $\mathrm{N}$ atoms further from the metal as in the case of $\mathrm{Rh}$ and $\mathrm{Ru}$. Another common observation is the large variation in adsorption energies depending on the employed exchange-correlation approximation. For instance, Laskowski et al. report adsorption energies of $-0.27,-0.19$, and $-0.04 \mathrm{eV} / \mathrm{BN}$ with LDA, WC-GGA, and PBE, respectively.

We carried out geometry optimizations of $h$-BN on $\mathrm{Ni}$ considering all six possible high-symmetry registries. The $\mathrm{Ni}(111)$ slab of seven $6 \times 6$ layers is symmetrically terminated on both sides by a $6 \times 6 h$-BN layer. Since the lattice constants match quite precisely, no large deformations of the $h$-BN layer are expected upon optimization. The calculated adsorption energies and work functions, as well as some structural parameters obtained in different registries are summarized in Table 9. The strongest interaction is obtained when the $\mathrm{N}$ atoms are atop surface atoms. In this case also, the distance from the substrate is significantly shorter than in structures where the $\mathrm{N}$ is located at the hollow sites. The differences among all other registries are much less pronounced, although when both $\mathrm{B}$ and $\mathrm{N}$ occupy hollow sites the distance from the substrate is slightly larger, the buckling smaller, and the work function larger. Furthermore, we observe a reduction in the surface magnetic moment from 0.65 to $0.50 \mu_{\mathrm{B}}$ as the interlayer distance decreases, the last value being the equilibrium result. Compared to the magnetic moment of the free $\mathrm{Ni}(111)$ surface, $0.73 \mu_{B}$, this corresponds to a reduction of -0.08 to $-0.23 \mu_{\mathrm{B}}$, in good agreement with previously published values (cf. Table 8 ). The magnetic moment of the innermost metal layer (bulk-like), however, 
Table 8 Adsorption energy [eV/BN], layer separation and corrugation $[\AA]$, and change in magnetic moment of the surface Ni atoms $\left[\mu_{\mathrm{B}}\right]$ upon adsorption of the $h$-BN overlayer on $\mathrm{Ni}(111)$ at different registries

\begin{tabular}{|c|c|c|c|c|c|c|}
\hline Method & $\mathrm{XC}$ & $E_{\text {ads }}$ & $d_{\mathrm{m}} i n(\mathrm{~B})$ & $b_{\mathrm{B}-\mathrm{N}}$ & $\Delta \mu_{\text {surf }}$ per $\mathrm{Ni}$ & References \\
\hline DFT & PBE-GGA & - & 2.08 & 0.11 & -0.08 & {$[20]$} \\
\hline \multirow[t]{2}{*}{ DFT } & PW91-GGA & $-0.021-0.038$ & $2.18-3.97$ & - & -0.00 to -0.23 & {$[25]$} \\
\hline & LDA & -0.420 to -0.141 & $1.95-3.11$ & - & -0.02 to -0.43 & {$[25]$} \\
\hline \multirow[t]{3}{*}{ DFT } & PBE-GGA & -0.04 & 2.04 & 0.11 & -0.06 & {$[21]$} \\
\hline & WC-GGA & -0.19 & 2.03 & 0.11 & & {$[21]$} \\
\hline & LDA & -0.27 & 2.01 & 0.11 & & {$[21]$} \\
\hline DFT & PBE-GGA & -0.04 & 1.99 & 0.10 & - & {$[74]$} \\
\hline DFT & $\operatorname{rev} \mathrm{PBE}+\mathrm{D} 3$ & -0.392 & 2.008 & 0.124 & -0.08 to -0.23 & Present work \\
\hline LEED & & - & 2.20 & 0.2 & - & {$[77]$} \\
\hline XPD & & - & 1.95 & 0.07 & - & {$[76]$} \\
\hline
\end{tabular}

Table 9 Adsorption and interaction energies [eV/BN], work function $\Phi[\mathrm{eV}]$, and distances, corrugation and buckling $[\AA]$ in the $h$-BN/Ni(111) system, using a $6 \times 6$ symmetric slab model in the six registries listed

\begin{tabular}{llllllllll}
\hline $\mathrm{N}$ & $\mathrm{B}$ & $E_{\text {ads }}$ & $E_{\text {int }}$ & $\Phi$ & $d_{\min }(\mathrm{B})$ & $d_{\min }(\mathrm{N})$ & $\Delta h_{\mathrm{BN}}$ & $b_{\mathrm{B}-\mathrm{N}}$ & $\mu_{\text {surf }}$ per Ni \\
\hline top & fcc & -0.390 & -0.470 & 3.58 & 2.006 & 2.130 & 0.001 & 0.123 & 0.519 \\
top & hcp & -0.392 & -0.473 & 3.55 & 2.008 & 2.132 & 0.001 & 0.124 & 0.503 \\
fcc & top & -0.283 & -0.257 & 3.84 & 2.903 & 2.921 & 0.010 & 0.022 & 0.635 \\
hcp & top & -0.283 & -0.268 & 3.84 & 2.923 & 2.944 & 0.057 & 0.021 & 0.630 \\
fcc & hcp & -0.285 & -0.269 & 3.90 & 3.015 & 3.028 & 0.028 & 0.016 & 0.651 \\
hcp & fcc & -0.293 & -0.270 & 3.91 & 2.996 & 3.008 & 0.073 & 0.016 & 0.649 \\
\hline
\end{tabular}

remains unchanged with a magnetic moment of about $0.58 \mu_{\mathrm{B}}$, irrespective of the monolayer-surface distance.

In the most stable registries, our results agree well with the published experimental values of interlayer distance and corrugation, being between the values determined from LEED and XPD. The distance between $h$-BN and Ni(111) and the corrugation of the overlayer also agree well with most previous computational studies [20, 21, 74] (cf. Table 8 ). The computed adsorption energies indicate also in the case of $\mathrm{Ni}(111)$ that the configurations with $\mathrm{N}$ atoms atop the surface $\mathrm{Ni}$ atoms are most stable. It is, however, not possible to definitively distinguish between B-fcc and B-hcp due to the very small differences in adsorption energy. $h$-BN turns out to be chemisorbed on $\mathrm{Ni}(111)$ in all six registries, with generally significantly stronger adsorption energies than previous studies have shown. This effect can be attributed to the contribution from the vdW correction, which was not considered in previous studies.

Figure 8 contains the spin-polarized PDOS projected on the $\mathrm{Ni}$ and $\mathrm{N}$ atoms in the most strongly bound registry, (N-top,B-fcc). The left column shows curves in the bulk and the surface atoms of the $h$-BN/Ni system and those at the bare Ni surface for comparison. The most pronounced change in the PDOS around the Fermi level upon the adsorption of $h$-BN occurs in the $d_{x z, y z}$ and $d_{z^{2}}$ states, while the orbitals without a $z$ component remain largely unaffected. For all orbitals, we observe a pronounced peak in the spin $\alpha$ channel just below the Fermi level and at or beyond it in the $\beta$-PDOS. Upon adsorption, these peaks shift to lower and higher energies in the $d_{z^{2}}$ and $d_{x z}, y z$ orbitals, respectively.

In the right column of Fig. 8, the electronic states are projected on the $\mathrm{N} p$ orbitals. We tried to adjust energy reference of the PDOS from the free-standing to one from the adsorbed $h$-BN by aligning the low-energy peaks, but when the peaks in the $p_{x, y}$ match well, in $p_{z}$ the main peaks are about $1 \mathrm{eV}$ higher in energy in the free-standing $h$-BN; this indicates a different response in the two different kinds of $p$ orbitals to the adsorption. Interestingly, the largest peaks more than $3 \mathrm{eV}$ below the Fermi energy in the spin $\alpha$ and $\beta p_{z}$ PDOS lie at the same energies, differences appearing only close and above the Fermi energy. Compared to a free-standing $h$-BN sheet, a number of weak gap states appears upon the adsorption, mainly originating from $p_{z}$. The shape of the curves remains otherwise quite similar, indicating that the electronic structure of the adsorbate is affected only weakly by the adsorption. We also note a small spin polarization of the PDOS, shifting the $\beta$ channel to slightly higher energies, and some differences in gap states between the two spins. 

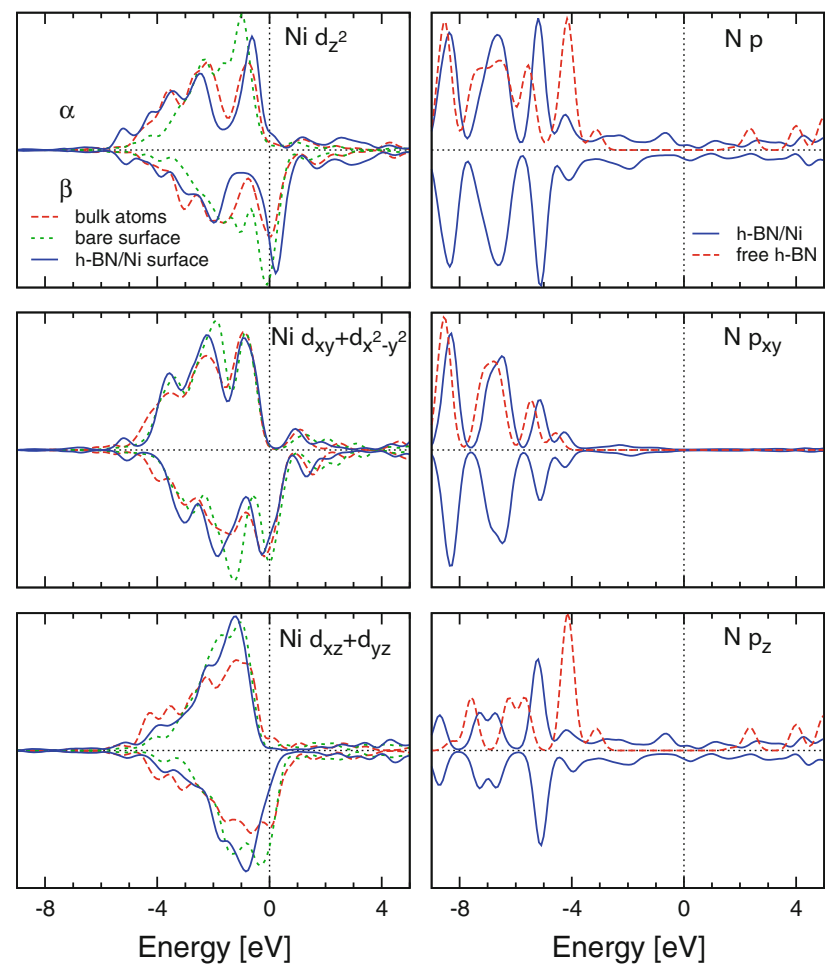

Fig. 8 PDOS on Ni $d$ states and $\mathrm{N} p$ states in the $h$-BN/Ni(111) system. States of spin $\alpha$ and $\beta$ are plotted on the positive and negative vertical axis, respectively. PDOS of a free, non-spin-polarized $h$-BN sheet is shown for comparison. Plots for Ni show projections on the surface and central atoms of the adsorbate system and of the bare surface for comparison. Energy scale is relative to the Fermi energy, in the free $h$-BN layer aligned at the peaks in PDOS at deep energies

Several related investigations of the density of states of $h$-BN/Ni have been published recently $[21,23,74]$. Our results are in good agreement with these reports. In particular, our data reproduce the approximate peak shape and position of the Ni $d_{z^{2}}$ states reported in [21], both at the surface with $h$-BN ( 3 peaks) and the bare surface ( 2 peaks). The relative shifts of the peaks closest to the Fermi level upon adsorption are also comparable to those results. Concerning the nitrogen states, the small spin polarization that we find (discussed below) matches the results in Ref. [21]. Che and Cheng [74] found similar peaks in the $\mathrm{N} p_{z}$ states, only sharper and more pronounced than ours.

Complementary to the PDOS plots, maps of the electron and spin density differences are plotted in Fig. 9 in the same system. Evidently, the electronic changes upon adsorption are predominantly confined to the $h$-BN monolayer and the topmost layer of metal atoms, while the lower-lying $\mathrm{Ni}$ atoms are largely inert except for a weak polarization in the second layer. The electron density difference indicates a transfer of electrons from the $\mathrm{Ni} d_{z^{2}}$ orbitals to the $d_{x z, y z}$ orbitals and to the interlayer space. At the same time, the $\mathrm{N} p_{z}$ orbitals lose electron density, whereas the $p_{x, y}$ gain some. An opposite effect occurs on the $\mathrm{B}$ atoms, which accumulate electrons in the $p_{z}$ orbitals and lose some from the in-plane orbitals. The B- $p_{z}$ orbital gets strongly polarized towards the metal surface. The image also implies a depletion of electrons at the centre of the $h$-BN honeycomb.

With regard to spin density, the most significant change of magnetism occurs-as expected-in the topmost $\mathrm{Ni}$ layer. The spin polarization in the $d_{z^{2}}$-symmetry orbitals increases, while spin polarization decreases in the $d_{x z, y z}$ orbitals. Some decrease in spin polarization is also visible in the second Ni layer. More notably, however, the nitrogen atoms are also subject to some magnetic polarization, albeit much smaller in magnitude. In particular, the average magnetic moment (from Mulliken population analysis) of the $\mathrm{N}$ atoms is $0.027 \mu_{\mathrm{B}}$ while that of $\mathrm{B}$ is $-0.020 \mu_{\mathrm{B}}$. These observations are consistent with the PDOS results discussed above. The upshift of the peak in the
Fig. 9 Map of the differences upon the adsorption of $h$-BN in total electron density (left) and spin density (right) of the $h$-BN/ $\mathrm{Ni}(111)$ system. Blue regions indicate depletion of electrons (or magnetic moment), while red areas denote accumulation

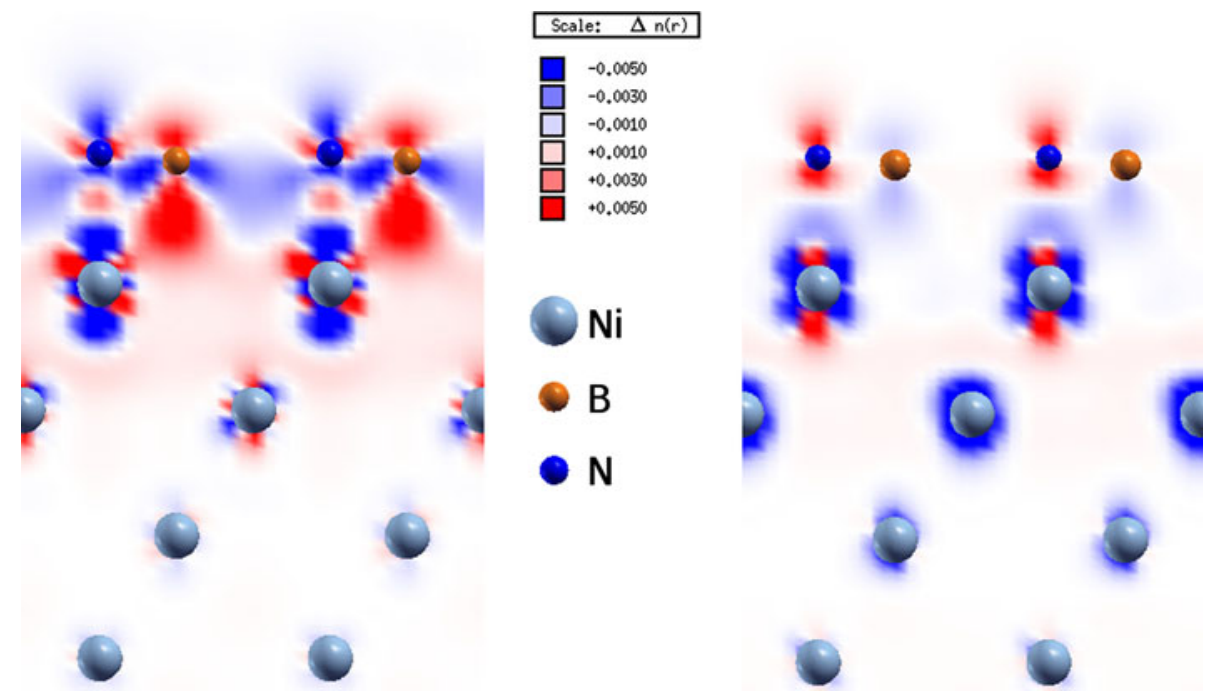




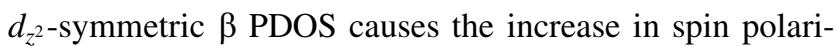
zation of the corresponding orbitals, while the move of $d_{x z}$, $y z$ orbitals to lower energies leads to the loss of spin polarization in these directions.

\section{Summary}

In this overview, we have shown that DFT calculations, when carefully set up, can be used to reproduce and explain experimental findings for the nanomesh systems. Our specific approach based on the Gaussian and plane waves method provides a similar accuracy of description as plane wave-based codes. Additionally, we apply a dispersion correction to semi-local density functionals.

The adsorption of $h$ - BN on the various substrates leads to different characteristics of the overlayer structure as summarized in Table 10. The strength of the interaction of $h$-BN with the metals in quasi-commensurate cases-either in real systems of $\mathrm{Ni}$ and $\mathrm{Cu}$ or in artificial $\mathrm{Rh}^{*}$ system when the lattice constant of the overlayer is scaled to match the substrate-follows the expected trend, being larger in the case of the transition metals and weaker on the noble metal $\mathrm{Cu}$. Whether the geometry of the overlayer is flat or buckled is mainly dictated by the lattice mismatch between $h$-BN and the substrate. On $\mathrm{Ru}(0001)$ and $\mathrm{Rh}(111)$, the mismatch is large and the interaction in the preferred regions strong. Therefore, an incommensurability is induced that is relieved by forming a corrugated nanomesh, where various lateral registries are found within the unit cell. On the other hand, on $\mathrm{Ni}(111)$, the mismatch is very small so that the commensurate $(1 \times 1)$ registry is obtained in experiments, with no corrugation. On $\mathrm{Cu}(111)$, the interaction is weak and the mismatch small, resulting in Moiré structures with large periodicities [73]. For all the investigated systems, we have employed dispersion corrections to the DFT functional. These corrections are

Table 10 General properties in the different $h$-BN/TM and nanomesh systems: mismatch between the equilibrium hexagonal lattice constants between the $h$-BN and the substrate [\%], corrugation $[\AA]$, adsorption energy and interaction energy $[\mathrm{eV} / \mathrm{BN}]$, smallest vertical TM-N and TM-B distances $[\AA]$, and the share of atoms forming the pore region (closer than $2.4 \AA$ on $\mathrm{Rh}$ and $2.2 \AA$ on $\mathrm{Ru}$ ) [\%]

\begin{tabular}{llllllll}
\hline & Mismatch & $\Delta h_{\mathrm{N}}$ & $E_{\text {ads }}$ & $E_{\text {int }}$ & $d_{\min }(\mathrm{N})$ & $d_{\min }(\mathrm{B})$ & $\begin{array}{l}\text { In- } \\
\text { pore }\end{array}$ \\
\hline $\mathrm{Ni}$ & +0.5 & - & -0.39 & -0.47 & 2.13 & 2.07 & - \\
$\mathrm{Cu}$ & -2.2 & 0.02 & -0.27 & -0.30 & 2.98 & 2.97 & - \\
$\mathrm{Ru}$ & -7.6 & 1.5 & -0.38 & -0.54 & 2.19 & 2.08 & 46 \\
$\mathrm{Rh}$ & -7.0 & 1.1 & -0.25 & -0.31 & 2.21 & 2.13 & 20 \\
$\mathrm{Rh}^{*}$ & 0.0 & - & - & -0.62 & 2.61 & & - \\
\hline
\end{tabular}

$\mathrm{Rh}^{*}$ refers to the system where the lattice constant of the $h$-BN was artificially scaled to match the hexagonal in-plane value of the $\mathrm{Rh}(111)$ essential to reproduce reliable structures, in particular when the interaction is weak and the distance between overlayer and substrate is large.

In order to better understand the nature of the interaction between $h$-BN and the four substrates, we have considered how the electronic structure of the overlayer is modified. $\mathrm{On} \mathrm{Rh}$ and $\mathrm{Ru}$, the regions where good contact is possible are characterized by strong re-hybridization of the $p$-states on the $\mathrm{N}$ atoms. In particular, we observe that these areas are more extended on $\mathrm{Ru}$, where the 14-on-13 reconstruction turns out to be the most favourable. Like previous studies, our calculations yield the magnetization of the $h$-BN overlayer on $\mathrm{Ni}(111)$. This surface magnetization could lead to applications including spin filtering, as adsorbates on this system would experience a weak, mainly insulating but yet spin-polarized interaction with the $h$-BN/ $\mathrm{Ni}(111)$. Our results confirm that in the spectrum of $h$-BN$\mathrm{TM}$ electronic interaction strength, $\mathrm{Cu}$ resides at the very low end and shows the weakest effects on $h$-BN of all metals discussed in this study.

Having established reliability and accuracy of the computational method for the overlayer arrangement of $h$-BN on metal supports, we can go further and use this approach not only to verify and explain experimental findings but also to predict new arrangements and trends of similar nanostructures and for example adsorption and assembly of molecules on them. The first examples have already appeared in the literature: Disappearance of the corrugation in the nanomesh on $\mathrm{Rh}(111)$ upon hydrogen intercalation [18] and water interacting with the nanomesh $[64,65,68]$.

Acknowledgments This work is supported by Swiss National Science Foundation under Grants No. CRSI20 122703 and No. 140441. The authors thank the Swiss National Supercomputer Centre (CSCS) and University of Zurich for generous allocations of computer time.

Open Access This article is distributed under the terms of the Creative Commons Attribution License which permits any use, distribution, and reproduction in any medium, provided the original author(s) and the source are credited.

\section{References}

1. Corso M, Auwärter W, Muntwiler M, Tamai A, Greber T, Osterwalder J (2004) Boron nitride nanomesh. Science 303(5655):217-220

2. Berner S, Corso M, Widmer R, Groening O, Laskowski R, Blaha P, Schwarz K, Goriachko A, Over H, Gsell S, Schreck M, Sachdev H, Greber T, Osterwalder J (2007) Boron nitride nanomesh: functionality from a corrugated monolayer. Angew Chem Int Ed 46(27):5115-5119

3. Goriachko A, He Y, Knapp M, Over H, Corso M, Brugger T, Berner S, Osterwalder J, Greber T (2007) Self-assembly of a hexagonal boron nitride nanomesh on $\mathrm{Ru}(0001)$. Langmuir 23(6):2928-2931 
4. Preobrajenski AB, Nesterov MA, Ng ML, Vinogradov AS, Martensson N (2007) Monolayer $h$-BN on lattice-mismatched metal surfaces: on the formation of the nanomesh. Chem Phys Lett 446(1-3):119-123

5. Brugger T, Guenther S, Wang B, Dil JH, Bocquet ML, Osterwalder J, Wintterlin J, Greber T (2009) Comparison of electronic structure and template function of single-layer graphene and a hexagonal boron nitride nanomesh on $\mathrm{Ru}(0001)$. Phys Rev B 79(4):045407

6. Cavar E, Westerstrom R, Mikkelsen A, Lundgren E, Vinogradov AS, Ng ML, Preobrajenski AB, Zakharov AA, Martensson N (2008) A single $h$-BN layer on Pt(111). Surf Sci 602(9):1722-1726

7. Preobrajenski AB, Vinogradov AS, Ng ML, Cavar E, Westerstrom R, Mikkelsen A, Lundgren E, Martensson N (2007) Influence of chemical interaction at the lattice-mismatched $h$-BN/ $\mathrm{Rh}(111)$ and $h$-BN/Pt(111) interfaces on the overlayer morphology. Phys Rev B 75(24):245412

8. Preobrajenski AB, Vinogradov AS, Martensson N (2005) Monolayer of $h$-BN chemisorbed on $\mathrm{Cu}(111)$ and $\mathrm{Ni}(111)$ : the role of the transition metal 3d states. Surf Sci 582(1-3):21-30

9. Preobrajenski AB, Krasnikov SA, Vinogradov AS, Ng ML, Kaambre T, Cafolla AA, Martensson N (2008) Adsorption-induced gap states of $h$-BN on metal surfaces. Phys Rev B 77(8):085412

10. Shimoyama I, Baba Y, Sekiguchi T, Nath KG (2009) A theoretical interpretation of near edge $\mathrm{X}$-ray absorption fine structure of hexagonal boron nitride monolayer on $\mathrm{Ni}(111)$. J Electron Spectrosc 175(1-3):6-13

11. Morscher M, Corso M, Greber T, Osterwalder J (2006) Formation of single layer $h$-BN on Pd(111). Surf Sci 600(16):3280-3284

12. Müller F, Hüfner S, Sachdev H, Laskowski R, Blaha P, Schwarz K (2010) Epitaxial growth of hexagonal boron nitride on Ag(111). Phys Rev B 82(11):113406

13. Müller F, Hüfner S, Sachdev H (2008) One-dimensional structure of boron nitride on chromium (110) - a study of the growth of boron nitride by chemical vapour deposition of borazine. Surf Sci 602(22):3467-3476

14. Vinogradov NA, Zakharov AA, Ng ML, Mikkelsen A, Lundgren $\mathrm{E}$, Mårtensson N, Preobrajenski $\mathrm{AB}$ (2012) One-dimensional corrugation of the $h$-BN monolayer on $\mathrm{Fe}(110)$. Langmuir 28(3): $1775-81$

15. Allan M, Berner S, Corso M, Greber T, Osterwalder J (2007) Tunable self-assembly of one-dimensional nanostructures with orthogonal directions. Nanoscale Res Lett 2(2):94-99

16. Wintterlin J, Bocquet ML (2009) Graphene on metal surfaces. Surf Sci 603(10-12):1841-1852

17. Dil H, Lobo-Checa J, Laskowski R, Blaha P, Berner S, Osterwalder J, Greber T (2008) Surface trapping of atoms and molecules with dipole rings. Science 319(5871):1824-1826

18. Brugger T, Ma HF, Iannuzzi M, Berner S, Winkler A, Hutter J, Osterwalder J, Greber T (2010) Nanotexture switching of singlelayer hexagonal boron nitride on rhodium by intercalation of hydrogen atoms. Angew Chem Int Ed 49(35):6120-6124

19. Laskowski R, Blaha P, Gallauner T, Schwarz K (2007) Singlelayer model of the hexagonal boron nitride nanomesh on the Rh(111) surface. Phys Rev Lett 98(10):106802

20. Grad G, Blaha P, Schwarz K, Auwärter W, Greber T (2003) Density functional theory investigation of the geometric and spintronic structure of $h$ - BN/Ni(111) in view of photoemission and STM experiments. Phys Rev B 68(8):085404

21. Laskowski R, Blaha P, Schwarz K (2008) Bonding of hexagonal $\mathrm{BN}$ to transition metal surfaces: an ab initio density-functional theory study. Phys Rev B 78(4):045,409

22. Laskowski R, Blaha $P$ (2008) Unraveling the structure of the $h$-BN/Rh(111) nanomesh with ab initio calculations. J Phys Condens Matt 20(6), 2nd workshop on theory meets industry, Erwin Schrodinger Inst, Vienna, Austria, 12-14 Jun 2007
23. Laskowski R, Gallauner T, Blaha P, Schwarz K (2009) Density functional theory simulations of B K and N K NEXAFS spectra of $h$-BN/transition metal(111) interfaces. J Phys Condens Matt 21(10): 104210

24. Laskowski R, Blaha P (2010) Ab initio study of $h$-BN nanomeshes on $\operatorname{Ru}(001), \operatorname{Rh}(111)$, and Pt(111). Phys Rev B 81(7):075418

25. Huda MN, Kleinman L (2006) $h$-BN monolayer adsorption on the Ni(111) surface: a density functional study. Phys Rev B 74(7):075418

26. Yazyev OV, Pasquarello A (2010) Metal adatoms on graphene and hexagonal boron nitride: towards rational design of selfassembly templates. Phys Rev B 82(4): 045407

27. Wang B, Bocquet ML (2011) Monolayer Graphene and $h$-BN on metal substrates as versatile templates for metallic nanoclusters. J Phys Chem Lett 2(18):2341-2345

28. Zhou YG, Zu XT, Gao F (2011) Substrate-induced magnetism in BN layer: a first-principles study. Solid State Commun 151(12):883-886

29. Lippert G, Hutter J, Parrinello M (1997) A hybrid Gaussian and plane wave density functional scheme. Mol Phys 92(3):477-487

30. VandeVondele J, Krack M, Mohamed F, Parrinello M, Chassaing T, Hutter J (2005) QUICKSTEP: fast and accurate density functional calculations using a mixed Gaussian and plane waves approach. Comput Phys Commun 167(2):103-128

31. Grimme S (2004) Accurate description of van der Waals complexes by density functional theory including empirical corrections. J Comput Chem 25(12):1463-1473

32. Grimme S, Antony J, Ehrlich S, Krieg H (2010) A consistent and accurate ab initio parametrization of density functional dispersion correction (DFT-D) for the 94 elements H-Pu. J Chem Phys 132(15): 154104

33. Hohenberg P, Kohn W (1964) Inhomogeneous electron gas. Phys Rev 136:B864-B871

34. Kohn W, Sham LJ (1965) Self-consistent equations including exchange and correlation effects. Phys Rev 140:A1133-A1138

35. CP2K (2012) CP2K developers group under the terms of the GNU General Public Licence; see http://www.cp2k.org/

36. Goedecker S, Teter M, Hutter J (1996) Separable dual-space Gaussian pseudopotentials. Phys Rev B 54(3):1703-1710

37. Hartwigsen C, Goedecker S, Hutter J (1998) Relativistic separable dual-space Gaussian pseudopotentials from $\mathrm{H}$ to $\mathrm{Rn}$. Phys Rev B 58(7):3641-3662

38. Krack M (2005) Pseudopotentials for $\mathrm{H}$ to $\mathrm{Kr}$ optimized for gradient-corrected exchange-correlation functionals. Theor Chem Acc 114(1-3): 145-152

39. Perdew JP, Burke K, Ernzerhof M (1996) Generalized gradient approximation made simple. Phys Rev Lett 77:3865-3868

40. Perdew JP, Burke K, Ernzerhof M (1997) Erratum: generalized gradient approximation made simple. Phys Rev Lett 78:1396

41. Zhang Y, Yang W (1998) Generalized gradient approximation made simple. Phys Rev Lett 80:890-891

42. Bendt P, Zunger A (1982) New approach for solving the densityfunctional self-consistent-field problem. Phys Rev B 26:3114

43. Marzari N, Vanderbilt D (1997) Maximally localized generalized Wannier functions for composite energy bands. Phys Rev B $56: 12847-12865$

44. Tersoff J, Hamann DR (1983) Theory and application for the scanning tunnelling microscope. Phys Rev Lett 50:1998-2001

45. Tersoff J, Hamann DR (1985) Theory of the scanning tunnelling microscope. Phys Rev B 31:805-813

46. VandeVondele J, Hutter J (2007) Gaussian basis sets for accurate calculations on molecular systems in gas and condensed phases. J Chem Phys 127(11):114105

47. Gonze X, Rignanese G, Verstraete M, Beuken J, Pouillon Y, Caracas R, Jollet F, Torrent M, Zerah G, Mikami M, Ghosez P, Veithen M, Raty J, Olevano V, Bruneval F, Reining L, Godby R, Onida G, Hamann D, Allan D (2005) A brief introduction to the ABINIT software package. Z Kristallogr 220(5-6):558-562 
48. Gonze X, Amadon B, Anglade PM, Beuken JM, Bottin F, Boulanger P, Bruneval F, Caliste D, Caracas R, Cote M, Deutsch T, Genovese L, Ghosez P, Giantomassi M, Goedecker S, Hamann DR, Hermet P, Jollet F, Jomard G, Leroux S, Mancini M, Mazevet S, Oliveira MJT, Onida G, Pouillon Y, Rangel T, Rignanese GM, Sangalli D, Shaltaf R, Torrent M, Verstraete MJ, Zerah G, Zwanziger JW (2009) ABINIT: first-principles approach to material and nanosystem properties. Comput Phys Commun 180(12):2582-2615

49. Giannozzi P, Baroni S, Bonini N, Calandra M, Car R, Cavazzoni C, Ceresoli D, Chiarotti GL, Cococcioni M, Dabo I, Dal Corso A, de Gironcoli S, Fabris S, Fratesi G, Gebauer R, Gerstmann U, Gougoussis C, Kokalj A, Lazzeri M, Martin-Samos L, Marzari N, Mauri F, Mazzarello R, Paolini S, Pasquarello A, Paulatto L, Sbraccia C, Scandolo S, Sclauzero G, Seitsonen AP, Smogunov A, Umari P, Wentzcovitch RM (2009) QUANTUM ESPRESSO: a modular and open-source software project for quantum simulations of materials. J Phys Condens Matt 21(39):395502

50. Blöchl PE (1994) Projector augmented-wave method. Phys Rev B 50(24):17953-17979

51. Owen E, Yates E, Sully A (1937) An X-ray investigation of pure iron-nickel alloys. Part 4: the variation of lattice-parameter with composition. P Phys Soc 49(Part 1):315-322

52. Straumanis ME, Yu LS (1969) Lattice parameters, densities, expansion coefficients and perfection of structure of $\mathrm{Cu}$ and of $\mathrm{Cu}-\mathrm{In} \alpha$ phase. Acta Cryst A 25(6):676-682

53. Finkel V, Kovtun G, Palatnik M (1971) X-ray-diffraction study of thermal expansion of ruthenium, osmium and rhenium at 77-300 degrees K. Phys Met Metallogr-USSR 32(1):231

54. Singh HP (1968) Determination of thermal expansion of Germanium, Rhodium and Iridium by X-rays. Acta Cryst A A 24(Part 4):469

55. Asato M, Settels A, Hoshino T, Asada T, Blugel S, Zeller R, Dederichs P (1999) Full-potential KKR calculations for metals and semiconductors. Phys Rev B 60(8):5202-5210

56. Ozolins V, Korling M (1993) Full-potential calculations using the generalized gradient approximation-structural-properties of transition metals. Phys Rev B 48(24):18304-18307

57. Vitos L, Ruban A, Skriver H, Kollar J (1998) The surface energy of metals. Surf Sci 411(1-2):186-202

58. Michaelson HB (1977) The work function of the elements and its periodicity. J Appl Phys 48(11):4729-4733

59. Furthmüller J, Hafner J, Kresse G (1994) Ab-initio calculation of the structural and electronic properties of carbon and boronnitride using ultrasoft pseudopotentials. Phys Rev B 50(21): 15606-15622

60. Wyckoff RWG (1965) Crystal structures, 2nd edn, vol 3. Interscience Publishers, New York

61. Pease RS (1952) An X-ray study of boron nitride. Acta Cryst 5(3):356
62. Kobayashi K, Watanabe K, Taniguchi T (2007) First-principles study of various hexagonal BN phases. J Phys Soc Jpn 76(10):104707

63. Müller F, Hüfner S, Sachdev H (2009) Epitaxial growth of boron nitride on a $\mathrm{Rh}(111)$ multilayer system: formation and fine tuning of a BN-nanomesh. Surf Sci 603(3):425-432

64. Ding Y, Iannuzzi M, Hutter J (2011a) Investigation of boron nitride nanomesh interacting with water. J Phys Chem C 115(28):13685-13692

65. Ding Y, Iannuzzi M, Hutter J (2011b) Investigation of $h$-BN/ $\mathrm{Rh}$ (111) nanomesh interacting with water and atomic hydrogen. Chimia 65(4):256-259

66. Koch HP, Laskowski R, Blaha P, Schwarz K (2011) Adsorption of gold atoms on the $\mathrm{h}-\mathrm{BN} / \mathrm{Rh}(111)$ nanomesh. Phys Rev B 84(24): 155404

67. Venkataramanan NS, Khazaei M, Sahara R, Mizuseki H, Kawazoe Y (2009) First-principles study of hydrogen storage over $\mathrm{Ni}$ and Rh-doped BN sheets. Chem Phys 359(1-3):173-178

68. Ma H, Brugger T, Berner S, Ding Y, Iannuzzi M, Hutter J, Osterwalder J, Greber T (2010) Nano-ice on boron nitride nanomesh: accessing proton disorder. ChemPhysChem 11(2):399-403

69. Stradi D, Barja S, Diaz C, Garnica M, Borca B, Hinarejos JJ, Sanchez-Portal D, Alcami M, Arnau A, Vazquez de Parga AL, Miranda R, Martin F (2011) Role of dispersion forces in the structure of graphene monolayers on Ru surfaces. Phys Rev Lett 106(18): 186102

70. Goriachko A, He YB, Over H (2008) Complex growth of nanoAu on $\mathrm{BN}$ nanomeshes supported by $\mathrm{Ru}(0001)$. J Phys Chem $\mathrm{C}$ 112(22):8147-8152

71. Martoccia D, Brugger T, Bjoerck M, Schlepuetz CM, Pauli SA, Greber T, Patterson BD, Willmott PR (2010) $h$-BN/Ru(0001) nanomesh: a 14-on-13 superstructure with $3.5 \mathrm{~nm}$ periodicity. Surf Sci 604(5-6):L16-L19

72. Wu Z, Cohen RE (2006) More accurate generalized gradient approximation for solids. Phys Rev B 73:235116

73. Joshi S, Ecija D, Koitz R, Iannuzzi M, Seitsonen AP, Hutter J, Sachdev H, Vijayaraghavan S, Bischoff F, Seufert K, Barth J, Auwärter W (2012) Hexagonal Boron Nitride on $\mathrm{Cu}(111)$ : An electronically corrugated monolayer. Nano Lett 12:5821-5828

74. Che JG, Cheng HP (2005) First-principles investigation of a monolayer of $\mathrm{C}_{60}$ on $h$-BN/Ni(111). Phys Rev B 72:115436

75. Nagashima A, Tejima N, Gamou Y, Kawai T, Oshima C (1995a) Electronic dispersion relations of monolayer hexagonal boron nitride formed on the Ni(111) surface. Phys Rev B 51(7): 4606-4613

76. Muntwiler M, Auwärter W, Baumberger F, Hoesch M, Greber T (2001) Determining adsorbate structures from substrate emission X-ray photoelectron diffraction. Surf Sci 472:125-132

77. Nagashima A, Tejima N, Gamou Y, Kawai T, Oshima C (1995b) Electronic structure of monolayer hexagonal boron nitride physisorbed on metal surfaces. Phys Rev Lett 75(21):3918-3921 